\title{
Godunov scheme and sampling technique for computing phase transitions in traffic flow modeling
}

\author{
Christophe CHALONS ${ }^{\dagger}$ \\ Université Denis Diderot-Paris 7 \& Laboratoire Jacques-Louis Lions, UMR 7598, \\ Université Pierre et Marie Curie-Paris 6, F-75005 Paris, France \\ PAOLA GOATIN \\ Institut de Mathématiques de Toulon et du Var, \\ I.S.I.T.V., Université du Sud Toulon-Var, \\ B.P. 56, F-83162 La Valette du Var Cedex, France
}

[Received 20 November 2006 and in revised form 26 November 2007]

\begin{abstract}
A new version of Godunov scheme is proposed in order to compute solutions of a traffic flow model with phase transitions. The scheme is based on a modified averaging strategy and a sampling procedure. Several numerical tests are shown to prove the validity of the method. The convergence of the algorithm is demonstrated numerically. We also give a higher order extension of the method in space and time.
\end{abstract}

Keywords: Hyperbolic conservation laws; continuous traffic models; Godunov scheme; phase transitions; sampling.

\section{Introduction}

We are interested in the numerical approximation of the solutions of a continuous traffic flow model taking into account the phenomenon of phase transitions between a free and a congested environment. The model we consider has been proposed by Colombo [10]. It consists of a scalar conservation law describing the free flow, and of a $2 \times 2$ system of conservation laws when the flow is congested. The coupling is achieved by introducing phase transitions between the free and the congested phase. We recall that, from the analytical point of view, the model is well posed for all initial data with bounded total variation [11].

Other traffic flow models with phase transitions have been considered in the literature since the 60-ties, in order to explain empirical flow-density relations (see Helbing [15, Section II] for description of the features recovered by a detailed analysis of the fundamental diagram). In particular, we refer the reader to the scalar model of Drake, Schofer and May [12]. Another model has been introduced recently by the second author [13].

Historically, one of the first continuous models introduced to describe traffic flow is the well known Lighthill-Whitham [19] and Richards [20] (LWR) model, which reads

$$
\partial_{t} \rho+\partial_{x}[\rho v(\rho)]=0,
$$

\footnotetext{
†E-mail: chalons@math.jussieu.fr

‡E-mail: goatin@univ-tln.fr
} 
where $\rho \in[0, R]$ is the mean traffic density, and $v(\rho)$, the mean traffic velocity, is a given nonincreasing function, non-negative for $\rho$ between 0 and the positive maximal density $R$, which corresponds to a traffic jam. This scalar model expresses conservation of the number of cars, and relies on the assumption that the car speed depends only on the density (more complex closure relations between speed and density, involving the density gradient, can be assumed: see [2] and references therein). This phenomenological relation is valid in steady state conditions, and is not realistic in more complicated situations. In particular, as shown in Figure 1, the corresponding fundamental diagram in the $(\rho, \rho v)$-plane does not qualitatively match experimental data at high densities.
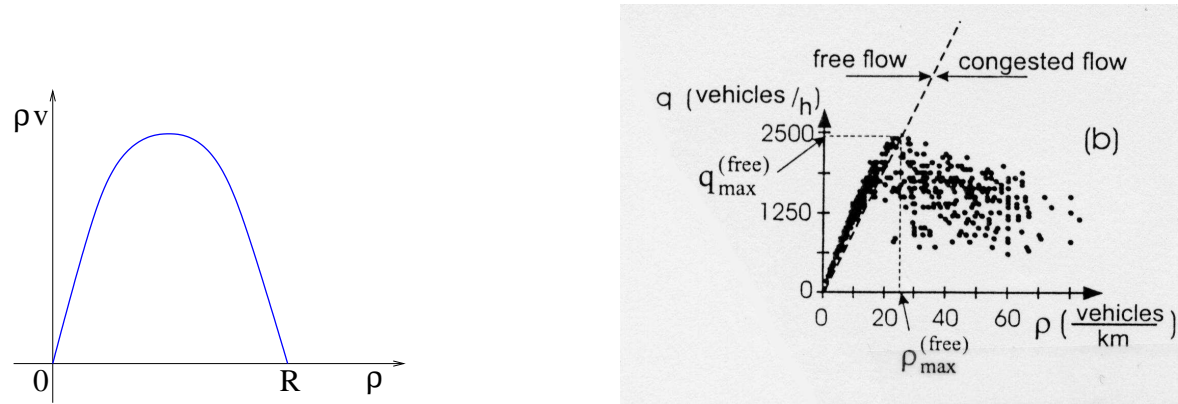

FIG. 1. Left: standard flow for the LWR model. Right: experimental data, taken from [17]; here $q$ denotes the flux $\rho v$.

The diagram above suggests that a good traffic flow model should exhibit two qualitatively different behaviors:

- for low densities, the flow is free and essentially analogous to the LWR model;

- at high densities the flow is congested and covers a 2-dimensional domain in the fundamental diagram; a "second order" model seems more appropriate to describe this dynamic.

From a numerical point of view, the presence of phase transitions makes standard numerical schemes useless. For example, it is easy to see that the classical Godunov method is not applicable due to the lack of convexity of the whole model phase space. Indeed, the latter turns out to be a disconnected set in $\mathbb{R}^{2}$, made of two connected components associated with the free and the congested domains, respectively. In the presence of phase transitions, the projection step taking place in the classical Godunov method can then give values which are not in the domain. This necessarily stops the procedure. We are thus led to propose a new version of the Godunov method, based on a modified averaging strategy and a sampling procedure. More precisely, we modify the mesh cells following the phase boundaries, so that the projection involves only values belonging to the same phase. In order to come back to the original cells, we complete the projection step with a Glimm-type sampling technique.

This scheme is essentially first order accurate, and hence introduces a considerable dissipation away from phase transitions. In order to improve accuracy, we also present an extension of the method to second-order accuracy in space and time, which is $L^{1}$-stable in space.

We remark that the techniques presented here apply also to models in [12, 13].

The averaging procedure on modified cells that we introduce has first been used (up to our knowledge) in [24] but in a different context and in a slightly different form. However, the idea 
of going back to the initial cells by means of a sampling procedure is new and allows us to avoid dealing with moving meshes (as in [24]). It has been motivated by recent work by the first author on approximating nonclassical solutions arising in certain nonlinear hyperbolic equations (see [4], [5] and the references therein), and very recently by Chalons and Coquel in [6] on computing sharp discrete shock profiles. Let us underline that the model studied here differs a lot from the one addressed in [24], since we are coupling systems of different dimensions. Moreover, we describe a higher order strategy adapted to our model.

The paper is organized as follows. In Section 2 we present the model, whose Riemann solver is described in Section 3. Section 4 is devoted to the description of the modified Godunov scheme and its higher order extension, which are tested in Section 5. Finally, Section 6 is devoted to the computation of the conservation errors.

\section{Governing equations}

We describe here the traffic flow model introduced by Colombo [10].

For the free flow, the well known LWR scalar conservation law is used,

$$
\partial_{t} \rho+\partial_{x}\left(\rho v_{f}(\rho)\right)=0, \quad x \in \mathbb{R}, t>0,
$$

where $\rho$ is the car density and $\rho \mapsto v_{f}(\rho)$ is the speed function given by

$$
v_{f}(\rho)=V\left(1-\frac{\rho}{R}\right)
$$

When the traffic is congested, the model is a $2 \times 2$ system of conservation laws [9]:

$$
\left\{\begin{array}{l}
\partial_{t} \rho+\partial_{x}\left(\rho v_{c}(\rho, q)\right)=0, \\
\partial_{t} q+\partial_{x}\left((q-Q) v_{c}(\rho, q)\right)=0,
\end{array} \quad x \in \mathbb{R}, t>0,\right.
$$

where $\rho$ still denotes the car density, $q$ is the weighted linear momentum and $Q$ is a parameter depending on the road under consideration and is related to the phenomenon of wide moving jams. The speed law now reads

$$
v_{c}(\rho, q)=\left(1-\frac{\rho}{R}\right) \frac{q}{\rho} .
$$

In (3) and (5), $R$ and $V$ are constants, the maximal possible car density and the maximal possible speed respectively.

It is worth noticing that one may provide the free system with a natural value for $q$ when setting $q=\rho V$. Indeed, $v_{f}(\rho)=v_{c}(\rho, \rho V)$, so that the associated conservation law is obtained by multiplying (2) by $V$ and reads

$$
\partial_{t} q+\partial_{x}\left(q v_{f}(\rho)\right)=0, \quad q=\rho V,
$$

or equivalently

$$
\partial_{t} q+\partial_{x}\left(q v_{c}(\rho, q)\right)=0, \quad q=\rho V .
$$

Recall that for the congested phase, $q$ evolves according to

$$
\partial_{t} q+\partial_{x}\left((q-Q) v_{c}(\rho, q)\right)=0 .
$$


This means that $q$ is not conserved across a phase transition. On the contrary, $\rho$ is conserved, as we will see in Section 3

The phase space $\Omega_{f}$ (resp. $\Omega_{c}$ ) for the free system (resp. the congested system) is chosen to be an invariant set for (2) (resp. (4)). (We refer the reader to [16] for definition and properties of invariant sets.) This implies that if the initial data are entirely in the free (resp. congested) phase, then the solution will belong to the free (resp. congested) phase for all time. The domains are chosen to be

$$
\begin{aligned}
\Omega_{f} & =\left\{(\rho, q) \in[0, R] \times\left[0,+\infty\left[: v_{f}(\rho) \geqslant V_{f}, q=\rho V\right\}\right.\right. \\
& =\left\{(\rho, q) \in[0, R] \times\left[0,+\infty\left[: \rho / R \leqslant 1-V_{f} / V, q=\rho V\right\}\right.\right.
\end{aligned}
$$

and

$$
\Omega_{c}=\left\{(\rho, q) \in[0, R] \times\left[0,+\infty\left[: 0 \leqslant v_{c}(\rho, q) \leqslant V_{c}, \frac{Q^{-}-Q}{R} \leqslant \frac{q-Q}{\rho} \leqslant \frac{Q^{+}-Q}{R}\right\},\right.\right.
$$

where $V_{f}>V_{c}$ are threshold speeds such that above $V_{f}$ the flow is free and below $V_{c}$ it is congested. Notice that the strict inequality $V_{f}>V_{c}$ is necessary to have uniqueness of solutions. In addition, $\left.Q^{-} \in\right] 0, Q\left[\right.$ and $\left.Q^{+} \in\right] Q,+\infty[$ depend on the environmental conditions and determine the width of the congested phase.

We introduce the following shortened form:

$$
\partial_{t} \mathbf{u}+\partial_{x} \mathbf{f}(\mathbf{u})=0, \quad \mathbf{u} \in \Omega=\Omega_{f} \cup \Omega_{c},
$$

for the model of phase transitions under consideration, with

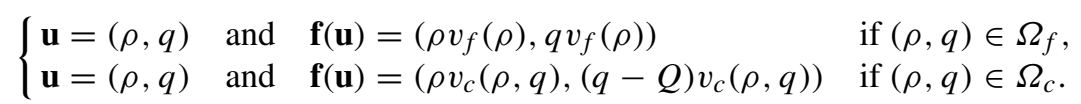

In the forthcoming developments, it is important to keep in mind that $\mathbf{u}$ and $\mathbf{f}(\mathbf{u})$ do not have the same meaning in the free phase and in the congested phase. Moreover, the domain $\Omega$ is not connected, hence it is not convex (see Figure2, left, in Section 3 .

To conclude this section, let us underline that $(8)$ is now supplemented, as is customary, with a given value of the solution at time $t=0$. More precisely, we assume that given an initial data $\mathbf{u}_{0} \in \Omega$ we have

$$
\mathbf{u}(\cdot, t=0)=\mathbf{u}_{0} .
$$

\section{The Riemann problem}

We recall in this section the description of the Riemann solver for (8)-(9), i.e. the self-similar solution of the Cauchy problem

$$
\left\{\begin{array}{l}
\partial_{t} \mathbf{u}+\partial_{x} \mathbf{f}(\mathbf{u})=0 \\
\mathbf{u}_{0}(x)= \begin{cases}\mathbf{u}^{l} & \text { if } x<0 \\
\mathbf{u}^{r} & \text { if } x>0\end{cases}
\end{array}\right.
$$

We refer the reader to [10] for the definition of admissible and consistent solutions to [10]. In particular, we recall that the Rankine-Hugoniot condition must hold at the phase transition:

$$
\rho(\Lambda t+, t) v_{c}(\mathbf{u}(\Lambda t+, t))-\rho(\Lambda t-, t) v_{f}(\rho(\Lambda t-, t))=\Lambda(\rho(\Lambda t+, t)-\rho(\Lambda t-, t))
$$


for all $t>0$, where $x=\Lambda t$ is the location of the phase transition. Notice that this condition ensures that the total number of cars is conserved.

Before giving a detailed description of the solutions to 10 , we recall the basic features of models (2) and (4). In the free phase the characteristic speed is $\lambda(\rho)=V(1-2 \rho / R)$, while the information on system (4) is collected in the following table:

$$
\begin{array}{ll}
r_{1}(\rho, q)=\left[\begin{array}{c}
\rho \\
q-Q
\end{array}\right], & r_{2}(\rho, q)=\left[\begin{array}{c}
R-\rho \\
\frac{R}{\rho} q
\end{array}\right], \\
\lambda_{1}(\rho, q)=\left(\frac{2}{R}-\frac{1}{\rho}\right) \cdot(Q-q)-\frac{Q}{R}, & \lambda_{2}(\rho, q)=v_{c}(\rho, q), \\
\nabla \lambda_{1} \cdot r_{1}(\rho, q)=2 \frac{Q-q}{R}, & \nabla \lambda_{2} \cdot r_{2}(\rho, q)=0, \\
\mathcal{L}_{1}\left(\rho ; \rho_{0}, q_{0}\right)=Q+\frac{q_{0}-Q}{\rho_{0}} \rho, & \mathcal{L}_{2}\left(\rho ; \rho_{0}, q_{0}\right)=\frac{\rho}{\rho_{0}} \frac{R-\rho_{0}}{R-\rho} q_{0}, \\
w_{1}(\rho, q)=v_{c}(\rho, q), & w_{2}(\rho, q)=\frac{q-Q}{\rho},
\end{array}
$$

where $r_{i}$ is the $i$-th right eigenvector, $\lambda_{i}$ the corresponding eigenvalue, $\mathcal{L}_{i}$ is the $i$-Lax curve and $w_{i}$ is the $i$-Riemann invariant. Shock and rarefaction curves coincide, hence system (4) belongs to the Temple class [21].

Using Riemann coordinates $\left(w_{1}, w_{2}\right)$, we have $\Omega_{c}=\left[0, V_{c}\right] \times\left[W_{2}^{-}, W_{2}^{+}\right]$. For $(\rho, q) \in \Omega_{f}$, we extend the corresponding Riemann coordinates $\left(w_{1}, w_{2}\right)$ as follows. Let $\tilde{\mathbf{u}}=(\tilde{\rho}, \tilde{\rho} V)$ be the point in $\Omega_{f}$ defined by $\tilde{\rho}=Q /\left(V-W_{2}^{-}\right)$. Define

$$
w_{1}=V_{f} \quad \text { and } \quad w_{2}= \begin{cases}V-Q / \rho & \text { if } \rho \geqslant \tilde{\rho}, \\ v_{f}(\tilde{\rho})-v_{f}(\rho)+V-Q / \tilde{\rho} & \text { if } \rho<\tilde{\rho},\end{cases}
$$

so that, in the Riemann coordinates, $\Omega_{f}=\left\{V_{f}\right\} \times\left[W_{0}, W_{2}^{+}\right]$(see Figure 2).


FIG. 2. Notations used in the paper.

All possible cases are listed below. Figures $3-5$ are plotted using the parameter values given in Section 5

(A) The data in $(10)$ are in the same phase, i.e. they are either both in $\Omega_{f}$ or both in $\Omega_{c}$. Then the solution is the standard Lax entropy solution to (2), resp. (4), and no phase boundary is present. 

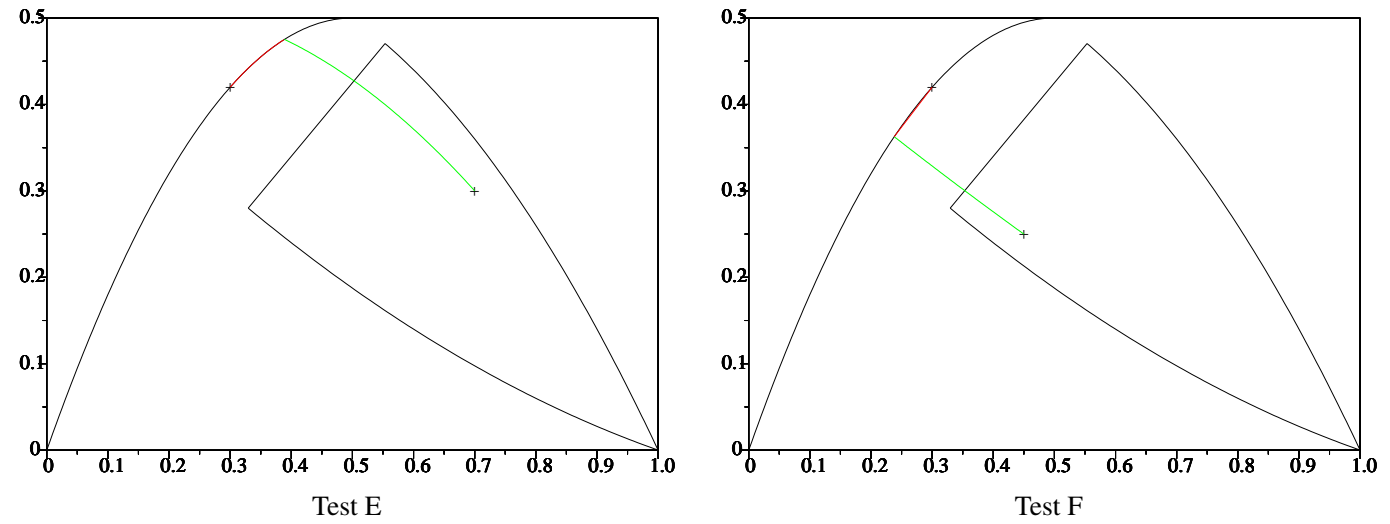

FIG. 3. Hugoniot loci in the phase plane $(\rho, \rho v)$ for case (B).

(B) $\mathbf{u}^{l} \in \Omega_{c}$ and $\mathbf{u}^{r} \in \Omega_{f}$ (as in Figure 3). We consider the points $\mathbf{u}^{c} \in \Omega_{c}$ and $\mathbf{u}^{m} \in \Omega_{f}$ implicitly defined by

$$
\begin{aligned}
\left(1-\frac{\rho^{c}}{R}\right)\left(Q+w_{2}\left(\mathbf{u}^{l}\right) \rho^{c}\right) & =\rho^{c} V_{c}, \\
\left(1-\frac{\rho^{m}}{R}\right)\left(Q+w_{2}\left(\mathbf{u}^{l}\right) \rho^{m}\right) & =\rho^{m} V\left(1-\frac{\rho^{m}}{R}\right) .
\end{aligned}
$$

If $w_{2}\left(\mathbf{u}^{l}\right)>0$, the solution is made of a 1-rarefaction from $\mathbf{u}^{l}$ to $\mathbf{u}^{c}$, a phase transition from $\mathbf{u}^{c}$ to $\mathbf{u}^{m}$ and a Lax wave from $\mathbf{u}^{m}$ to $\mathbf{u}^{r}$ (Section 5. Test E). If $w_{2}\left(\mathbf{u}^{l}\right) \leqslant 0$, we have a shock-like phase transition from $\mathbf{u}^{l}$ to $\mathbf{u}^{m}$ and a Lax wave from $\mathbf{u}^{m}$ to $\mathbf{u}^{r}$ (Section5. Test F).
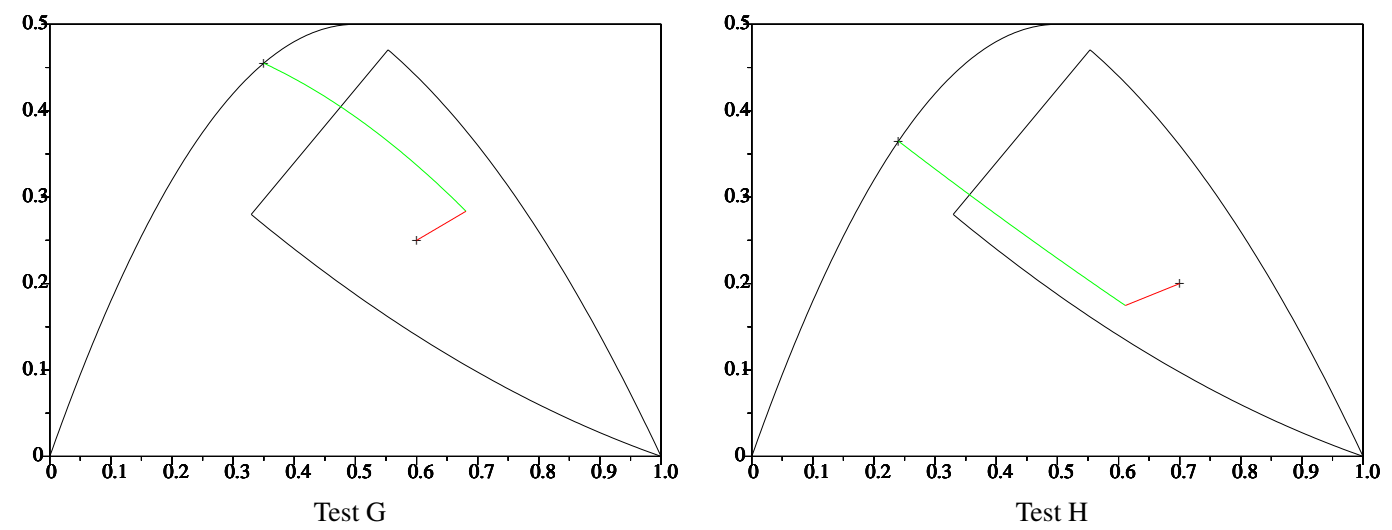

FIG. 4. Hugoniot loci in the phase plane $(\rho, \rho v)$ for case (C).

(C) $\mathbf{u}^{l} \in \Omega_{f}$ and $\mathbf{u}^{r} \in \Omega_{c}$ with $w_{2}\left(\mathbf{u}^{l}\right) \in\left[W_{2}^{-}, W_{2}^{+}\right]$(Figure 4 . Consider the points $\mathbf{u}^{c}$ and $\mathbf{u}^{m} \in \Omega_{c}$ implicitly defined by 


$$
\begin{aligned}
\left(1-\frac{\rho^{c}}{R}\right)\left(Q+w_{2}\left(\mathbf{u}^{l}\right) \rho^{c}\right) & =\rho^{c} V_{c}, \\
\left(1-\frac{\rho^{m}}{R}\right)\left(Q+w_{2}\left(\mathbf{u}^{l}\right) \rho^{m}\right) & =\rho^{m} w_{1}\left(\mathbf{u}^{r}\right) .
\end{aligned}
$$

If $w_{2}\left(\mathbf{u}^{l}\right)>0$, the solution is made of a shock-like phase transition from $\mathbf{u}^{l}$ to $\mathbf{u}^{m}$ and a 2contact discontinuity from $\mathbf{u}^{m}$ to $\mathbf{u}^{r}$ (Section 5 Test G). If $w_{2}\left(\mathbf{u}^{l}\right) \leqslant 0$, the solution displays a phase transition from $\mathbf{u}^{l}$ to $\mathbf{u}^{c}$, a 2-rarefaction from $\mathbf{u}^{c}$ to $\mathbf{u}^{m}$ and a 2-contact discontinuity from $\mathbf{u}^{m}$ to $\mathbf{u}^{r}$ (Section5. Test $\mathrm{H}$ ).


FIG. 5. Hugoniot loci in the phase plane $(\rho, \rho v)$ for case (D).

(D) $\mathbf{u}^{l} \in \Omega_{f}$ with $w_{2}\left(\mathbf{u}^{l}\right)<W_{2}^{-}$and $\mathbf{u}^{r} \in \Omega_{c}$ (see Figure 5. Let $\mathbf{u}^{m} \in \Omega_{c}$ be the point on the lower boundary of $\Omega_{c}$ implicitly defined by

$$
\left(1-\frac{\rho^{m}}{R}\right)\left(Q+W_{2}^{-} \rho^{m}\right)=\rho^{m} w_{1}\left(\mathbf{u}^{r}\right),
$$

and consider the speed of the phase boundary joining $\mathbf{u}^{l} \in \Omega_{f}$ to $\mathbf{u}^{m} \in \Omega_{c}$

$$
\Lambda\left(\mathbf{u}^{l}, \mathbf{u}^{m}\right)=\frac{\rho^{l} v_{f}\left(\rho^{l}\right)-\rho^{m} w_{1}\left(\mathbf{u}^{r}\right)}{\rho^{l}-\rho^{m}} .
$$

Let $\mathbf{U}_{c}=\left(R_{c}, Q_{c}\right) \in \Omega_{c}$ be the point whose Riemann coordinates are $\left(V_{c}, W_{2}^{-}\right)$. If $\lambda_{1}\left(\mathbf{U}_{c}\right) \geqslant$ $\Lambda\left(\mathbf{u}^{l}, \mathbf{U}_{c}\right)$, the solution is a phase transition from $\mathbf{u}^{l}$ to $\mathbf{U}_{c}$, a 1-rarefaction from $\mathbf{U}_{c}$ to $\mathbf{u}^{m}$ and a 2-contact discontinuity from $\mathbf{u}^{m}$ to $\mathbf{u}^{r}$. Otherwise:

- If $\lambda_{1}\left(\mathbf{u}^{m}\right) \leqslant \Lambda\left(\mathbf{u}^{l}, \mathbf{u}^{m}\right)$, the solution is a phase transition from $\mathbf{u}^{l}$ to $\mathbf{u}^{m}$ followed by a 2-contact discontinuity from $\mathbf{u}^{m}$ to $\mathbf{u}^{r}$ (Section 5 Test J).

- If $\lambda_{1}\left(\mathbf{u}^{m}\right)>\Lambda\left(\mathbf{u}^{l}, \mathbf{u}^{m}\right)$, let $\mathbf{u}^{c}=\left(\rho^{c}, q^{c}\right) \in \Omega_{c}$ be implicitly defined by

$$
\lambda_{1}\left(\mathbf{u}^{c}\right)=\Lambda\left(\mathbf{u}^{l}, \mathbf{u}^{c}\right),
$$

i.e. $\rho^{c}$ is the larger root of the equation

$$
\left(Q-Q^{-}\right) \rho^{2}-2 \rho^{l}\left(Q-Q^{-}\right) \rho+R^{2}\left(\rho^{l} v_{f}\left(\rho^{l}\right)-Q\right)+\rho^{l} R\left(2 Q-Q^{-}\right)=0
$$


and $q^{c}=Q-\rho^{c}\left(Q-Q^{-}\right) / R$. Then the solution shows a phase transition from $\mathbf{u}^{l}$ to $\mathbf{u}^{c}$, an attached 1-rarefaction from $\mathbf{u}^{c}$ to $\mathbf{u}^{m}$ and a 2-contact discontinuity from $\mathbf{u}^{m}$ to $\mathbf{u}^{r}$ (Section 5 . Test I).

\section{Numerical schemes}

Let us first introduce a space step $\Delta x$ and a time step $\Delta t$, both assumed to be constant for simplicity in the forthcoming developments. We set $v=\Delta t / \Delta x$. Then we define the mesh interfaces $x_{j+1 / 2}=$ $j \Delta x$ for $j \in \mathbb{Z}$ and the intermediate times $t^{n}=n \Delta t$ for $n \in \mathbb{N}$, and we seek at each time $t^{n}$ an approximation $\mathbf{u}_{j}^{n}$ of the solution of $8-(9)$ on the interval $\left[x_{j-1 / 2}, x_{j+1 / 2}\right), j \in \mathbb{Z}$. Therefore, a piecewise constant approximate solution $x \mapsto \mathbf{u}_{v}\left(x, t^{n}\right)$ of the solution $\mathbf{u}$ is given by

$$
\mathbf{u}_{v}\left(x, t^{n}\right)=\mathbf{u}_{j}^{n} \quad \text { for all } x \in \mathcal{C}_{j}=\left[x_{j-1 / 2}, x_{j+1 / 2}\right), j \in \mathbb{Z}, n \in \mathbb{N} .
$$

When $n=0$, we set $x_{j}=0.5 \cdot\left(x_{j-1 / 2}+x_{j+1 / 2}\right)$ and

$$
\mathbf{u}_{j}^{0}=\mathbf{u}_{0}\left(x_{j}\right) \quad \text { for all } j \in \mathbb{Z} .
$$

Note that the usual $L^{2}$-projection is not adapted in the present context since, depending on initial data, it could artificially introduce unphysical states which are not in the phase space at time $t=0$ (recall that $\Omega=\Omega_{f} \cup \Omega_{c}$ is not convex).

Given a sequence $\left(\mathbf{u}_{j}^{n}\right)_{j \in \mathbb{Z}}$ at time $t^{n}$, it is now a question of proposing a way of advancing it to the next time level $t^{n+1}$. Two manners of tackling that are presented. We begin with the celebrated Godunov scheme in its classical form and show that it is not relevant in the present setting of possible phase transitions. We are thus led to present a new version of this scheme based on a modified averaging strategy coupled with a sampling procedure. We also show an extension of this last method to second-order accuracy.

\subsection{Failure of the classical Godunov scheme}

As is well known, the Godunov scheme consists of two steps: the first step in which the initial data evolves in time according to the PDE model under consideration, and the second step of projection onto the piecewise constant functions. We review the procedure in detail in order to fix the notations.

Step 1: Evolution in time. In this first step, one solves the Cauchy problem

$$
\left\{\begin{array}{l}
\partial_{t} \mathbf{v}+\partial_{x} \mathbf{f}(\mathbf{v})=0, \quad x \in \mathbb{R} \\
\mathbf{v}(x, 0)=\mathbf{u}_{v}\left(x, t^{n}\right)
\end{array}\right.
$$

for times $t \in[0, \Delta t]$. Recall that $x \mapsto \mathbf{u}_{v}\left(x, t^{n}\right)$ is piecewise constant. Then, under the usual CFL restriction

$$
\frac{\Delta t}{\Delta x} \max _{\mathbf{v}}\left\{\left|\lambda_{i}(\mathbf{v})\right|: i=1 \text { if } \mathbf{v} \in \Omega_{f}, i=1,2 \text { if } \mathbf{v} \in \Omega_{c}\right\} \leqslant \frac{1}{2}
$$

for all the $\mathbf{v}$ under consideration, the solution of $\sqrt{13}$ is known by gluing together the solutions of the Riemann problems set at each interface. More precisely,

$$
\mathbf{v}(x, t)=\mathbf{v}_{\mathbf{r}}\left(\frac{x-x_{j+1 / 2}}{t} ; \mathbf{u}_{j}^{n}, \mathbf{u}_{j+1}^{n}\right) \quad \text { for all }(x, t) \in\left[x_{j}, x_{j+1}\right] \times[0, \Delta t],
$$


where $(x, t) \mapsto \mathbf{v}_{\mathbf{r}}\left(x / t ; \mathbf{v}^{l}, \mathbf{v}^{r}\right)$ denotes the self-similar solution of the Riemann problem

$$
\left\{\begin{array}{l}
\partial_{t} \mathbf{v}+\partial_{x} \mathbf{f}(\mathbf{v})=0, \quad x \in \mathbb{R}, t>0, \\
\mathbf{v}(x, 0)= \begin{cases}\mathbf{v}^{l} & \text { if } x<0, \\
\mathbf{v}^{r} & \text { if } x>0\end{cases}
\end{array}\right.
$$

whatever $\mathbf{v}^{l}$ and $\mathbf{v}^{r}$ are in the phase space $\Omega_{f} \cup \Omega_{c}$ (see also Section 3 above).

Step 2: Projection $\left(t^{n} \rightarrow t^{n+1}\right)$. The aim of this second step is to get a piecewise constant approximate solution on each cell $\mathcal{C}_{j}$ at time $t^{n+1}$. This may be simply done by averaging the solution $x \mapsto \mathbf{v}(x, \Delta t)$ given by [15), as expressed by the following update formula:

$$
\mathbf{u}_{j}^{n+1}=\frac{1}{\Delta x} \int_{x_{j-1 / 2}}^{x_{j+1 / 2}} \mathbf{v}(x, \Delta t) \mathrm{d} t, \quad j \in \mathbb{Z} .
$$

Actually, one can provide an even simpler formula for $\mathbf{u}_{j}^{n+1}$ by integrating equation 13 over the element $E=(a b c d)$ defined by $\left[x_{j-1 / 2}, x_{j+1 / 2}\right] \times[0, \Delta t]$ and represented in Figure 6 From Green's

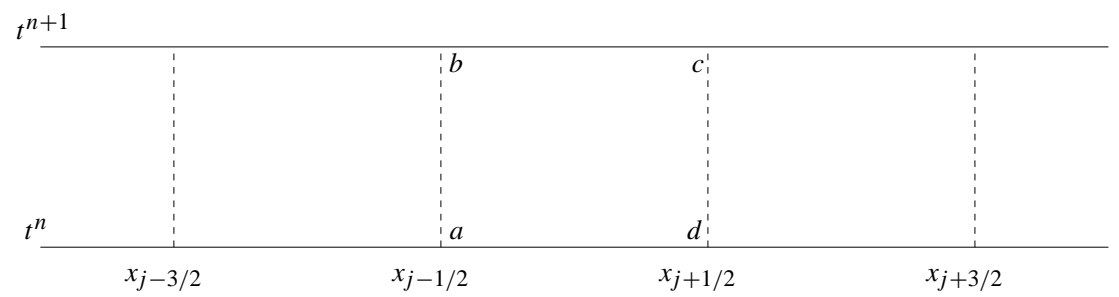

FIG. 6. Averaging element in the classical Godunov method.

theorem and using $(13)$ and $(16)$, we get

$$
\mathbf{u}_{j}^{n+1}=\mathbf{u}_{j}^{n}-\frac{\Delta t}{\Delta x}\left(\mathbf{f}_{j+1 / 2}^{n,-}-\mathbf{f}_{j-1 / 2}^{n,+}\right) \quad \text { for all } j \in \mathbb{Z},
$$

where the numerical fluxes are given by

$$
\mathbf{f}_{j+1 / 2}^{n, \pm}=\mathbf{f}\left(\mathbf{v}_{\mathbf{r}}\left(0^{ \pm} ; \mathbf{u}_{j}^{n}, \mathbf{u}_{j+1}^{n}\right)\right) \quad \text { for all } j \in \mathbb{Z},
$$

and classical notations have been used for the traces at $0^{-}$and $0^{+}$of the Riemann solutions under consideration.

To conclude the description of the classical Godunov scheme, observe that if $\mathbf{u}_{j}^{n}$ and $\mathbf{u}_{j+1}^{n}$ belong to the same phase (free or congested), the same holds for the Riemann solution $(x, t) \mapsto$ $\mathbf{v}_{\mathbf{r}}\left(x / t ; \mathbf{u}_{j}^{n}, \mathbf{u}_{j+1}^{n}\right)$ so that $\mathbf{v}_{\mathbf{r}}\left(0^{-} ; \mathbf{u}_{j}^{n}, \mathbf{u}_{j+1}^{n}\right)$ and $\mathbf{v}_{\mathbf{r}}\left(0^{+} ; \mathbf{u}_{j}^{n}, \mathbf{u}_{j+1}^{n}\right)$ are actually in the same phase. As a consequence, we necessarily have, for all $j \in \mathbb{Z}$,

$$
\mathbf{f}\left(\mathbf{v}_{\mathbf{r}}\left(0^{-} ; \mathbf{u}_{j}^{n}, \mathbf{u}_{j+1}^{n}\right)\right)=\mathbf{f}\left(\mathbf{v}_{\mathbf{r}}\left(0^{+} ; \mathbf{u}_{j}^{n}, \mathbf{u}_{j+1}^{n}\right)\right) .
$$

That is indeed obvious if $\mathbf{v}_{\mathbf{r}}\left(0^{-} ; \mathbf{u}_{j}^{n}, \mathbf{u}_{j+1}^{n}\right)=\mathbf{v}_{\mathbf{r}}\left(0^{+} ; \mathbf{u}_{j}^{n}, \mathbf{u}_{j+1}^{n}\right)$, while in the opposite case the associated discontinuity is necessarily stationary and $(18)$ follows from the Rankine-Hugoniot conditions. In other words, the method is conservative at the corresponding interface $j+1 / 2$. 
Otherwise, if $\mathbf{u}_{j}^{n}$ and $\mathbf{u}_{j+1}^{n}$ are not in the same phase, the states $\mathbf{v}_{\mathbf{r}}\left(0^{-} ; \mathbf{u}_{j}^{n}, \mathbf{u}_{j+1}^{n}\right)$ and $\mathbf{v}_{\mathbf{r}}\left(0^{+} ; \mathbf{u}_{j}^{n}, \mathbf{u}_{j+1}^{n}\right)$ may well be distinct and in two different phases. In such a situation, only the first components of $\mathbf{f}\left(\mathbf{v}_{\mathbf{r}}\left(0^{-} ; \mathbf{u}_{j}^{n}, \mathbf{u}_{j+1}^{n}\right)\right)$ and $\mathbf{f}\left(\mathbf{v}_{\mathbf{r}}\left(0^{+} ; \mathbf{u}_{j}^{n}, \mathbf{u}_{j+1}^{n}\right)\right)$ associated with the conservation of the mass in both the free and the congested system are equal. Indeed, recall that only the Rankine-Hugoniot relation coming from the mass conservation applies across a phase transition (see Section 3).

What is wrong with this method. Of course, the computation of the traces at $0^{-}$and $0^{+}$of the Riemann solvers at each interface $x_{j+1 / 2}$ does not represent a problem, since these solutions are known from Section 3 (see also [10]). Actually, the failure of this strategy is due to the lack of convexity of the domain $\Omega_{f} \cup \Omega_{c}$ in the $(\rho, q)$-plane (see Figure 2 and to the possible presence of phase transitions in the Riemann solutions. In this case, the state $\mathbf{u}_{j}^{n+1}$ resulting from the averaging procedure (16) may be outside $\Omega_{f} \cup \Omega_{c}$ for some $j \in \mathbb{Z}$ (even if the solution $\mathbf{u}_{v}\left(\cdot, t^{n}\right.$ ) belongs to the domain). This means that at the next time step, the Riemann solutions are generally not known (even not defined actually) at each interface $x_{j+1 / 2}$, so that the classical Godunov method stops. As already discussed by Zhong, Hou and LeFloch in [24], and even earlier by Abgrall [1] for simpler and more homogeneous problems, the problem we underline here is clearly located in the projection step of the Godunov method (Step 2). In order to know, everywhere in the domain and at each time step, in which phase the flow is, we now propose a modification of this second step.

\subsection{A new version of the Godunov scheme}

In this section, we present a new version of the Godunov scheme that will turn out to be more adapted to the model under consideration. We keep unchanged the first step of the method as it is described in the previous section. Indeed, let us recall that the failure of the classical Godunov method comes from the projection strategy proposed in the second step. The difference thus lies in the corresponding averaging procedure (16) that we are going to modify. The idea (see [24] in a different context) is to no longer average the solution $x \mapsto \mathbf{v}(x, \Delta t)$ on the mesh cells $\mathcal{C}_{j}=$ $\left[x_{j-1 / 2}, x_{j+1 / 2}\right.$ ), since they may contain states in different phases, but on (possibly) modified and non-uniform cells that we will denote $\overline{\mathcal{C}}_{j}^{n}=\left[\bar{x}_{j-1 / 2}^{n}, \bar{x}_{j+1 / 2}^{n}\right)$. These are constructed to contain values belonging to a single phase. To this end, the new cells are defined according to the position of the phase transitions. Then a sampling strategy will allow us to recover a piecewise constant solution on the initial mesh cells $\mathcal{C}_{j}$ (see also [4], [5] in a different context).

Step 2 (Modified): Projection $\left(t^{n} \rightarrow t^{n+1}\right)$. Let $\left(\sigma_{j+1 / 2}^{n}=\sigma\left(\mathbf{u}_{j}^{n}, \mathbf{u}_{j+1}^{n}\right)\right)_{j \in \mathbb{Z}}$ be a sequence of characteristic speeds of propagation at interfaces $\left(x_{j+1 / 2}\right)_{j \in \mathbb{Z}}$ such that:

- if $\mathbf{u}_{j}^{n}$ and $\mathbf{u}_{j+1}^{n}$ are not in the same phase (free or congested), then $\sigma_{j+1 / 2}^{n}$ coincides with the speed of propagation of the phase transition in the Riemann solution $(x, t) \mapsto \mathbf{v}_{\mathbf{r}}\left(x / t ; \mathbf{u}_{j}^{n}, \mathbf{u}_{j+1}^{n}\right)$,

- if $\mathbf{u}_{j}^{n}$ and $\mathbf{u}_{j+1}^{n}$ belong to the same phase, then $\sigma_{j+1 / 2}^{n}=0$.

Then, assuming that for all $j \in \mathbb{Z}$ the interface $x_{j+1 / 2}$ moves at velocity $\sigma_{j+1 / 2}^{n}$ between times $t^{n}$ and $t^{n+1}=t^{n}+\Delta t$, we define the new interface $\bar{x}_{j+1 / 2}^{n}$ at time $t^{n+1}$ setting

$$
\bar{x}_{j+1 / 2}^{n}=x_{j+1 / 2}+\sigma_{j+1 / 2}^{n} \Delta t, \quad j \in \mathbb{Z} .
$$

We also introduce

$$
\overline{\Delta x}_{j}^{n}=\bar{x}_{j+1 / 2}^{n}-\bar{x}_{j-1 / 2}^{n}, \quad j \in \mathbb{Z} .
$$


In particular and by definition of the sequence $\left(\sigma_{j+1 / 2}^{n}\right)_{j \in \mathbb{Z}}$, it is clear that on each modified cell $\overline{\mathcal{C}}_{j}^{n}=\left[\bar{x}_{j-1 / 2}^{n}, \bar{x}_{j+1 / 2}^{n}\right)$, the solution $x \mapsto \mathbf{v}(x, \Delta t)$ given by 15, is fully either in the free phase or in the congested phase. Then, averaging this solution on cells $\overline{\mathcal{C}}_{j}^{n}$ provides us with a piecewise constant approximate solution $\overline{\mathbf{u}}_{v}\left(x, t^{n+1}\right)$ on a non-uniform mesh defined by

$$
\overline{\mathbf{u}}_{v}\left(x, t^{n+1}\right)=\overline{\mathbf{u}}_{j}^{n+1} \quad \text { for all } x \in \overline{\mathcal{C}}_{j}^{n}, j \in \mathbb{Z}, n \in \mathbb{N},
$$

with

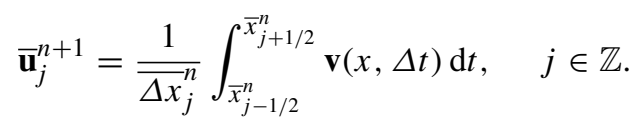

Let us underline that by the definition of the modified cells, we actually know which phase every constant state of the solution $\overline{\mathbf{u}}_{v}\left(x, t^{n+1}\right)$ belongs to. In fact, both $\Omega_{f}$ and $\Omega_{c}$ are convex domains (and so are stable under $L^{2}$-projection). This is the relevant difference from the solution $\mathbf{u}_{v}\left(x, t^{n+1}\right)$ obtained in the classical Godunov method, and justifies the new approach.

Let us notice that the modified cells $\overline{\mathcal{C}}_{j}^{n}$ may be either smaller or larger than the original ones $\mathcal{C}_{j}$, depending on the signs of the velocities $\sigma_{j+1 / 2}^{n}, j \in \mathbb{Z}$. This is illustrated in Figures 7 and 8

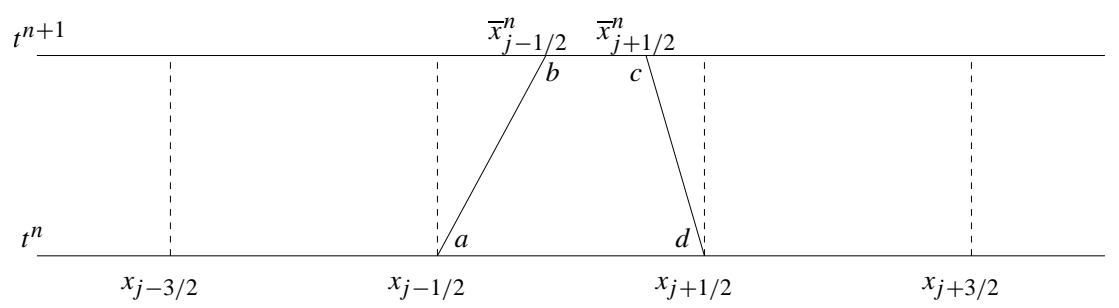

FIG. 7. A first example of averaging element in the modified Godunov method.

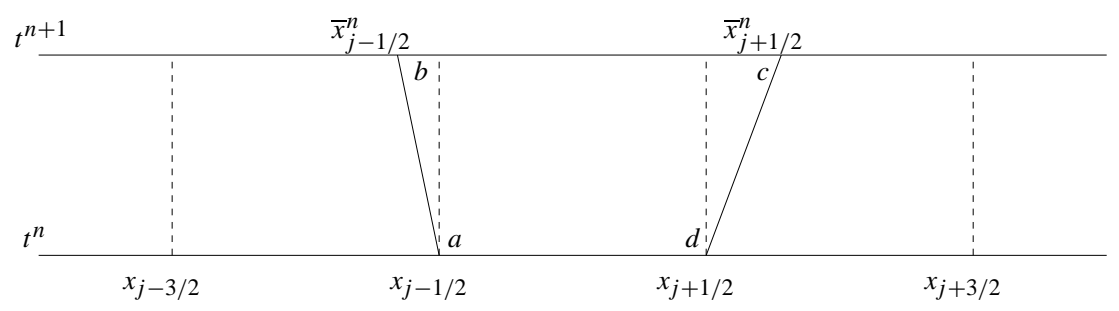

FIG. 8. A second example of averaging element in the modified Godunov method.

Even in this case, a simpler formula is obtained for $\overline{\mathbf{u}}_{j}^{n+1}$ by integrating equation $\sqrt{13}$ over the element $\bar{E}=(a b c d)$ defined by

$$
\bar{E}=\left\{(x, t): t \in[0, \Delta t] \text { and } x_{j-1 / 2}+\sigma_{j-1 / 2}^{n} t \leqslant x \leqslant x_{j+1 / 2}+\sigma_{j+1 / 2}^{n} t\right\}
$$

(see again Figures 7 and 8). Nevertheless, one has to be careful when applying Green's theorem owing to the fact that $E$ is no longer a rectangle. We get, using the same (usual) notations as in the 
previous section for the traces of the Riemann solutions at given points,

$$
\begin{aligned}
0= & \iint_{\bar{E}}\left[\partial_{t} \mathbf{v}+\partial_{x} \mathbf{f}(\mathbf{v})\right] \mathrm{d} x \mathrm{~d} t \\
= & \overline{\Delta x}_{j}^{n} \overline{\mathbf{u}}_{j}^{n+1}-\Delta x \mathbf{u}_{j}^{n}+\int_{0}^{\Delta t}\left[\mathbf{f}\left(\mathbf{v}_{\mathbf{r}}\left(\sigma_{j+1 / 2}^{n,-} ; \mathbf{u}_{j}^{n}, \mathbf{u}_{j+1}^{n}\right)\right)-\sigma_{j+1 / 2}^{n} \mathbf{v}_{\mathbf{r}}\left(\sigma_{j+1 / 2}^{n,-} ; \mathbf{u}_{j}^{n}, \mathbf{u}_{j+1}^{n}\right)\right] \mathrm{d} t \\
& -\int_{0}^{\Delta t}\left[\mathbf{f}\left(\mathbf{v}_{\mathbf{r}}\left(\sigma_{j-1 / 2}^{n,+} ; \mathbf{u}_{j-1}^{n}, \mathbf{u}_{j}^{n}\right)\right)-\sigma_{j-1 / 2}^{n} \mathbf{v}_{\mathbf{r}}\left(\sigma_{j-1 / 2}^{n,+} ; \mathbf{u}_{j-1}^{n}, \mathbf{u}_{j}^{n}\right)\right] \mathrm{d} t .
\end{aligned}
$$

Introducing now the numerical fluxes

$$
\overline{\mathbf{f}}_{j+1 / 2}^{n, \pm}=\mathbf{f}\left(\mathbf{v}_{\mathbf{r}}\left(\sigma_{j+1 / 2}^{n, \pm} ; \mathbf{u}_{j}^{n}, \mathbf{u}_{j+1}^{n}\right)\right)-\sigma_{j+1 / 2}^{n} \mathbf{v}_{\mathbf{r}}\left(\sigma_{j+1 / 2}^{n, \pm} ; \mathbf{u}_{j}^{n}, \mathbf{u}_{j+1}^{n}\right) \quad \text { for all } j \in \mathbb{Z},
$$

a condensed form, similar to 17 , is obtained for $\overline{\mathbf{u}}_{j}^{n+1}$ :

$$
\overline{\mathbf{u}}_{j}^{n+1}=\frac{\Delta x}{\overline{\Delta x}_{j}^{n}} \mathbf{u}_{j}^{n}-\frac{\Delta t}{\overline{\Delta x}_{j}^{n}}\left(\overline{\mathbf{f}}_{j+1 / 2}^{n,-}-\overline{\mathbf{f}}_{j-1 / 2}^{n,+}\right) \quad \text { for all } j \in \mathbb{Z} .
$$

At this stage, notice that if $\mathbf{u}_{j}^{n}$ and $\mathbf{u}_{j+1}^{n}$ are in the same phase, then $\mathbf{v}_{\mathbf{r}}\left(\sigma_{j+1 / 2}^{n,-} ; \mathbf{u}_{j}^{n}, \mathbf{u}_{j+1}^{n}\right)$ and $\mathbf{v}_{\mathbf{r}}\left(\sigma_{j+1 / 2}^{n,+} ; \mathbf{u}_{j}^{n}, \mathbf{u}_{j+1}^{n}\right)$ also does. The conservation property

$$
\begin{aligned}
\mathbf{f}\left(\mathbf{v}_{\mathbf{r}}\left(\sigma_{j+1 / 2}^{n,-} ; \mathbf{u}_{j}^{n}, \mathbf{u}_{j+1}^{n}\right)\right)-\sigma_{j+1 / 2}^{n} & \mathbf{v}_{\mathbf{r}}\left(\sigma_{j+1 / 2}^{n,-} ; \mathbf{u}_{j}^{n}, \mathbf{u}_{j+1}^{n}\right) \\
= & \mathbf{f}\left(\mathbf{v}_{\mathbf{r}}\left(\sigma_{j+1 / 2}^{n,+} ; \mathbf{u}_{j}^{n}, \mathbf{u}_{j+1}^{n}\right)\right)-\sigma_{j+1 / 2}^{n} \mathbf{v}_{\mathbf{r}}\left(\sigma_{j+1 / 2}^{n,+} ; \mathbf{u}_{j}^{n}, \mathbf{u}_{j+1}^{n}\right)
\end{aligned}
$$

then remains valid thanks to the Rankine-Hugoniot conditions. Actually, in such a situation $\sigma_{j+1 / 2}^{n}=0$ by definition. Otherwise, if $\mathbf{v}_{\mathbf{r}}\left(\sigma_{j+1 / 2}^{n,-} ; \mathbf{u}_{j}^{n}, \mathbf{u}_{j+1}^{n}\right)$ and $\mathbf{v}_{\mathbf{r}}\left(\sigma_{j+1 / 2}^{n,+} ; \mathbf{u}_{j}^{n}, \mathbf{u}_{j+1}^{n}\right)$ are not in the same phase, equality 22 makes sense only for the first component associated with the mass conservation.

Finally, we introduce the notation $\overline{\mathbf{f}}_{j+1 / 2}^{n, \pm}=\overline{\mathbf{f}}^{ \pm}\left(\mathbf{u}_{j}^{n}, \mathbf{u}_{j+1}^{n}\right)$ for the interface fluxes, with of course

$$
\overline{\mathbf{f}}^{ \pm}\left(\mathbf{u}_{j}^{n}, \mathbf{u}_{j+1}^{n}\right)=\mathbf{f}\left(\mathbf{v}_{\mathbf{r}}\left(\sigma_{j+1 / 2}^{n, \pm} ; \mathbf{u}_{j}^{n}, \mathbf{u}_{j+1}^{n}\right)\right)-\sigma_{j+1 / 2}^{n} \mathbf{v}_{\mathbf{r}}\left(\sigma_{j+1 / 2}^{n, \pm} ; \mathbf{u}_{j}^{n}, \mathbf{u}_{j+1}^{n}\right) .
$$

Recall that $\sigma_{j+1 / 2}^{n}=\sigma\left(\mathbf{u}_{j}^{n}, \mathbf{u}_{j+1}^{n}\right)$ by definition.

In order to avoid having to deal with moving meshes, we complete the projection step defining a new approximation $\mathbf{u}_{j}^{n+1}$ of the solution at time $t^{n+1}$ on the (uniform) cells $\mathcal{C}_{j}, j \in \mathbb{Z}$. To this end, for all $j \in \mathbb{Z}$, we propose to pick up randomly on the cell $\mathcal{C}_{j}$ a value $\overline{\mathbf{u}}_{j-1}^{n+1}, \overline{\mathbf{u}}_{j}^{n+1}$ or $\overline{\mathbf{u}}_{j+1}^{n+1}$, in agreement with their rate of presence in the cell. More precisely, given a well distributed random sequence $\left(a_{n}\right)$ within $(0,1)$, it amounts to setting

$$
\mathbf{u}_{j}^{n+1}= \begin{cases}\overline{\mathbf{u}}_{j-1}^{n+1} & \text { if } a_{n+1} \in\left(0, \frac{\Delta t}{\Delta x} \max \left(\sigma_{j-1 / 2}^{n}, 0\right)\right), \\ \overline{\mathbf{u}}_{j}^{n+1} & \text { if } a_{n+1} \in\left[\frac{\Delta t}{\Delta x} \max \left(\sigma_{j-1 / 2}^{n}, 0\right), 1+\frac{\Delta t}{\Delta x} \min \left(\sigma_{j+1 / 2}^{n}, 0\right)\right), \\ \overline{\mathbf{u}}_{j+1}^{n+1} & \text { if } a_{n+1} \in\left[1+\frac{\Delta t}{\Delta x} \min \left(\sigma_{j+1 / 2}^{n}, 0\right), 1\right),\end{cases}
$$

for all $j \in \mathbb{Z}$. Following a proposal by Collela [8], we consider the van der Corput random sequence $\left(a_{n}\right)$ defined by

$$
a_{n}=\sum_{k=0}^{m} i_{k} 2^{-(k+1)}
$$


where $n=\sum_{k=0}^{m} i_{k} 2^{k}, i_{k}=0,1$, denotes the binary expansion of the integer $n=1,2, \ldots$ For the sake of illustration, we easily find that the first few elements of this sequence are

$$
\begin{aligned}
& a_{1}=0.5, \quad a_{2}=0.25, \quad a_{3}=0.75, \quad a_{4}=0.125, \\
& a_{5}=0.625, \quad a_{6}=0.375, \quad a_{7}=0.875, \quad a_{8}=0.0625 \text {. }
\end{aligned}
$$

This sequence is actually well distributed within $(0,1)$. Moreover, one can prove for instance that $a_{i}<0.5$ for $i$ even and $a_{i}>0.5$ for $i$ odd. This well-known sequence is often favorite since, when used in the context of the Glimm scheme, it leads to very good results in the smooth parts of the solutions (see for instance $[8]$ and [7] for illustration). This concludes the description of the modified Godunov scheme.

We finish this section by emphasizing that due to the sampling procedure, the whole algorithm we propose (Step $1+$ Step 2 (Modified)) is not "strictly" conservative in the classical sense of finite volume methods. However, we numerically demonstrate in the next sections that it is actually "weakly" conservative in the following sense: first, phase transitions propagate with the right speeds (given by the Rankine-Hugoniot conditions) and then conservation errors seem to tend to zero with the mesh size.

REMARK. Of course, the random choice method (Glimm scheme) can be applied successfully to compute solutions of 8 . Nevertheless, our method does not require computing all the values in the Riemann solution, but only the values on both sides of the phase transition. Moreover, the algorithm coincides with the classical Godunov scheme, and hence it is conservative, away from phase transitions.

\subsection{Higher order extension of the method in space and time}

In this section, we describe a simultaneous space and time second-order extension of the new version of the Godunov scheme. Our strategy relies on the very popular MUSCL approach for the space accuracy and on a Runge-Kutta technique for the time accuracy. As usual, the second-order accuracy is obtained for smooth solutions only, even if better numerical results are also expected when discontinuities (or non-smooth regions) are present. In our context, it is important to notice that smooth solutions exist but necessarily remain in the same phase (free or congested) since phase changes are always associated with discontinuities. As a consequence, the resulting procedure has to be understood as second-order accurate away from phase transitions. This is the reason why we will focus on the first part of the projection step only, the sampling procedure being kept unchanged.

This section is organized as follows. We first address the space accuracy and show how to obtain a MUSCL scheme which is stable in the $L^{1}$ sense. Then we deal with the time accuracy and show how to apply a second-order Runge-Kutta technique.

Accuracy in space. We begin by briefly recalling the MUSCL method for obtaining the secondorder accuracy in space. For more details we refer the reader to [23], [14], [22] and the references therein, and also to a recent work by Berthon [3]. Assume that there exists a change of variables $\mathbf{u} \mapsto \mathbf{U}=\varphi(\mathbf{u})$ from $\Omega$ onto some set $\Omega_{\mathbf{U}}$. The starting point of the method consists in replacing at each time $t^{n}$ and on each cell $\mathcal{C}_{j}$ the constant values $\mathbf{u}_{j}^{n}$ by means of $\varphi$ and a linear reconstruction of $\mathbf{U}$. We set

$$
\left\{\begin{array}{l}
\mathbf{u}^{n}(x)=\varphi^{-1}\left(\mathbf{U}^{n}(x)\right), \quad x \in \mathcal{C}_{j}=\left[x_{j-1 / 2}, x_{j+1 / 2}\right), \quad \text { with } \\
\mathbf{U}^{n}(x)=\mathbf{U}_{j}^{n}+s_{j}^{n} \frac{x-x_{j}}{\Delta x}, \quad \mathbf{U}_{j}^{n}=\varphi\left(\mathbf{u}_{j}^{n}\right), \quad j \in \mathbb{Z} .
\end{array}\right.
$$


In $25, x_{j}$ represents the center of the cell $\mathcal{C}_{j}: x_{j}=\frac{1}{2}\left(x_{j-1 / 2}+x_{j+1 / 2}\right)$, and $s_{j}^{n}$ is the slope of the linear reconstruction. The choice of the reconstructed variable $\mathbf{U}$ generally depends on the system under consideration. In the present study, we will see below that in order to ensure the $L^{1}$-stability of the method, the reconstruction can be performed on the conservative variable for the free system $(\mathbf{U}=\mathbf{u})$, while the Riemann variables $\mathbf{U}=\left(w_{1}, w_{2}\right)$ turn out to be more adapted for the congested phase.

We denote by $\mathbf{u}_{j}^{n, \pm}$ and $\mathbf{U}_{j}^{n, \pm}$ the values at the edges $x=x_{j \pm 1 / 2}$ of $\mathbf{u}^{n}$ and $\mathbf{U}^{n}$ respectively:

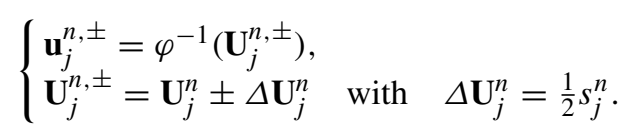

Then, following the basic principle of the MUSCL method, we propose to replace the couple $\left(\mathbf{u}_{j}^{n}, \mathbf{u}_{j+1}^{n}\right)$ with $\left(\mathbf{u}_{j}^{n,+}, \mathbf{u}_{j+1}^{n,-}\right)$ in the evaluation of the numerical fluxes $\overline{\mathbf{f}}_{j+1 / 2}^{n, \pm}$ at each interface $j+1 / 2$. More precisely, we now set $\overline{\mathbf{f}}_{j+1 / 2}^{n, \pm}=\overline{\mathbf{f}}^{ \pm}\left(\mathbf{u}_{j}^{n,+}, \mathbf{u}_{j+1}^{n,-}\right)$ instead of $\overline{\mathbf{f}}_{j+1 / 2}^{n, \pm}=\overline{\mathbf{f}}^{ \pm}\left(\mathbf{u}_{j}^{n}, \mathbf{u}_{j+1}^{n}\right)$ in 23 .

We now draw a particular attention to the choice of the reconstructed variable $\mathbf{U}$ and the slopes $s_{j}^{n}$. It is well-known that these have to be carefully determined for stability reasons. Once $\mathbf{U}$ is chosen, a usual choice for $s_{j}^{n}$, or equivalently for $\Delta \mathbf{U}_{j}^{n}$, is given by a slope-limiter procedure with for instance the so-called minmod limiter. It reads

$$
\Delta \mathbf{U}_{j}^{n}=\frac{1}{2} \operatorname{minmod}\left(\mathbf{U}_{j+1}^{n}-\mathbf{U}_{j}^{n}, \mathbf{U}_{j}^{n}-\mathbf{U}_{j-1}^{n}\right),
$$

where the minmod function is defined by

$$
\operatorname{minmod}(a, b)= \begin{cases}\operatorname{sign}(a) \min (|a|,|b|) & \text { if } a b \geqslant 0, \\ 0 & \text { otherwise, }\end{cases}
$$

for two scalar quantities $a$ and $b$ (sign denotes the sign function). In [27, minmod is applied componentwise.

REMARK. In the rest of this section, in order to avoid cumbersome notations, we will assume that the three states $\mathbf{U}_{j-1}^{n}, \mathbf{U}_{j}^{n}$ and $\mathbf{U}_{j+1}^{n}$ in $27 p$ belong to the same phase. Otherwise, if $\mathbf{U}_{j-1}^{n}$ and/or $\mathbf{U}_{j+1}^{n}$ are not in the same phase of $\mathbf{U}_{j}^{n}$, they are replaced in practice with $\varphi\left(\mathbf{u}_{+}\left(\mathbf{u}_{j-1}^{n}, \mathbf{u}_{j}^{n}\right)\right)$ and/or $\varphi\left(\mathbf{u}_{-}\left(\mathbf{u}_{j}^{n}, \mathbf{u}_{j+1}^{n}\right)\right)$, where $\mathbf{u}_{ \pm}\left(\mathbf{u}_{j}^{n}, \mathbf{u}_{j+1}^{n}\right)$ represent for all $j$ the values on both sides of the phase transition in the Riemann solution associated with the initial states $\mathbf{u}_{j}^{n}$ and $\mathbf{u}_{j+1}^{n}$. Then, by definition, $\varphi\left(\mathbf{u}_{+}\left(\mathbf{u}_{j-1}^{n}, \mathbf{u}_{j}^{n}\right)\right), \mathbf{u}_{j}^{n}$ and $\varphi\left(\mathbf{u}_{-}\left(\mathbf{u}_{j}^{n}, \mathbf{u}_{j+1}^{n}\right)\right)$ belong to the same phase.

Our objective is to enforce the $L^{1}$-stability of the reconstruction procedure in the sense that we want $\mathbf{u}_{j}^{n, \pm}$ to necessarily belong to the phase space $\Omega$. This stability property will be obtained by a relevant choice of $\mathbf{U}$ and after a possible correction of the "guess" increment defined by (27).

Let us begin with the case $\mathbf{u}_{j}^{n} \in \Omega_{f}$ and consider a conservative reconstruction: $\mathbf{U}=\mathbf{u}$. Since $q$ always equals $\rho V$ in the free phase, the constraints $\mathbf{u}_{j}^{n, \pm} \in \Omega_{f}$ read

$$
\left\{\begin{array}{l}
0 \leqslant \rho_{j}^{n, \pm} \leqslant R \\
V_{f} \leqslant v_{f}\left(\rho_{j}^{n, \pm}\right)
\end{array}\right.
$$


which by definition of $v_{f}$ is first equivalent to

$$
\left\{\begin{array}{l}
0 \leqslant \rho_{j}^{n, \pm} \leqslant R \\
\rho_{j}^{n, \pm} \leqslant R\left(1-V_{f} / V\right)
\end{array}\right.
$$

and then to

$$
0 \leqslant \rho_{j}^{n, \pm} \leqslant R\left(1-V_{f} / V\right)
$$

by positivity of $V_{f}$ and $V$. But $\rho_{j}^{n, \pm}=\rho_{j}^{n} \pm \Delta \rho_{j}^{n}$ so that a straightforward transformation shows that these two conditions read as follows on the increment $\Delta \rho_{j}^{n}$ :

$$
\left|\Delta \rho_{j}^{n}\right| \leqslant \min \left(\rho_{j}^{n}, R\left(1-V_{f} / V\right)-\rho_{j}^{n}\right) .
$$

Then, choosing the "guess" increment 27] under the constraint 287 immediately leads to the following definition of $\Delta \rho_{j}^{n}$ :

$\Delta \rho_{j}^{n}=\operatorname{sign}(\Delta \rho) \min \left(\rho_{j}^{n}, R\left(1-V_{f} / V\right)-\rho_{j}^{n}, \Delta \rho\right), \quad$ with $\Delta \rho=\frac{1}{2} \operatorname{minmod}\left(\rho_{j+1}^{n}-\rho_{j}^{n}, \rho_{j}^{n}-\rho_{j-1}^{n}\right)$.

We now turn to the case $\mathbf{u}_{j}^{n} \in \Omega_{c}$. The constraints $\mathbf{u}_{j}^{n, \pm} \in \Omega_{c}$ are equivalent to

$$
\left\{\begin{array}{l}
0 \leqslant w_{1}\left(\rho_{j}^{n, \pm}, q_{j}^{n, \pm}\right) \leqslant V_{c} \\
W_{2}^{-} \leqslant w_{2}\left(\rho_{j}^{n, \pm}, q_{j}^{n, \pm}\right) \leqslant W_{2}^{+}
\end{array}\right.
$$

Due to the nonlinearity of the two functions $w_{1}$ and $w_{2}$, we propose to perform the reconstruction on these variables (the Riemann coordinates) and to set $\mathbf{U}=\left(w_{1}, w_{2}\right)(\rho, q)$. Then choosing the corresponding increments $\Delta\left(w_{1}\right)_{j}^{n}$ and $\Delta\left(w_{2}\right)_{j}^{n}$ according to 277 yields

$$
\left\{\begin{array}{l}
\left(w_{1}\right)_{j}^{n, \pm}=\left(w_{1}\right)_{j}^{n} \pm \frac{1}{2} \operatorname{minmod}\left(\left(w_{1}\right)_{j+1}^{n}-\left(w_{1}\right)_{j}^{n},\left(w_{1}\right)_{j}^{n}-\left(w_{1}\right)_{j-1}^{n}\right), \\
\left(w_{2}\right)_{j}^{n, \pm}=\left(w_{2}\right)_{j}^{n} \pm \frac{1}{2} \operatorname{minmod}\left(\left(w_{2}\right)_{j+1}^{n}-\left(w_{2}\right)_{j}^{n},\left(w_{2}\right)_{j}^{n}-\left(w_{2}\right)_{j-1}^{n}\right),
\end{array}\right.
$$

which is easily seen to imply the last two constraints in 29 by definition of the minmod function (and since $\mathbf{u}_{j-1}^{n}$ and $\mathbf{u}_{j+1}^{n}$ are also assumed to be in $\Omega_{c}$ ). This shows that the "guess" choice 27 need not be modified in order to obtain the required $L^{1}$-stability property, provided that Riemann coordinates are used in the linear reconstruction procedure.

Accuracy in time. To conclude this section, we aim at proposing a time discretization which is second-order accurate in smooth regions and away from phase transitions. Of course, the definition of the strategy must take into account the presence of phase transitions. Actually, our objective is to propose a simple numerical time integration which is equivalent, away from phase transitions, to the well-known RK2 method (2nd order Runge-Kutta, or Heun). The reader is assumed to be familiar with the latter.

The MUSCL scheme obtained below reads

$$
\overline{\mathbf{u}}_{j}^{n+1}-\mathbf{u}_{j}^{n}=\frac{\Delta x-\overline{\Delta x}_{j}^{n}}{\overline{\Delta x}_{j}^{n}} \mathbf{u}_{j}^{n}-\frac{\Delta t}{\overline{\Delta x}_{j}^{n}}\left(\overline{\mathbf{f}}^{-}\left(\mathbf{u}_{j}^{n,+}, \mathbf{u}_{j+1}^{n,-}\right)-\overline{\mathbf{f}}^{+}\left(\mathbf{u}_{j-1}^{n,+}, \mathbf{u}_{j}^{n,-}\right)\right) .
$$

From this formula and the approximated values $\left(\mathbf{u}_{j}^{n}\right)_{j \in \mathbb{Z}}$ on the cells $\mathcal{C}_{j}$, we then define a first approximation $\overline{\mathbf{u}}_{j}^{n+1=}$ of the updated value on the cell $\overline{\mathcal{C}}_{j}^{n}$ by 


$$
\left.\overline{\mathbf{u}}_{j}^{n+1=}-\mathbf{u}_{j}^{n}=\frac{\Delta x-\overline{\Delta x}_{j}^{n}}{\overline{\Delta x}_{j}^{n}} \mathbf{u}_{j}^{n}-\frac{\Delta t}{\overline{\Delta x}_{j}^{n}} \overline{\mathbf{f}}^{-}\left(\mathbf{u}_{j}^{n,+}, \mathbf{u}_{j+1}^{n,-}\right)-\overline{\mathbf{f}}^{+}\left(\mathbf{u}_{j-1}^{n,+}, \mathbf{u}_{j}^{n,-}\right)\right) .
$$

Then another one denoted $\overline{\mathbf{u}}_{j}^{n+1-}$ is obtained from the first approximations $\left(\overline{\mathbf{u}}_{j}^{n+1=}\right)_{j \in \mathbb{Z}}$ :

$$
\overline{\mathbf{u}}_{j}^{n+1-}-\mathbf{u}_{j}^{n+1=}=\frac{\Delta x-\overline{\Delta x}_{j}^{n}}{\overline{\Delta x}_{j}^{n}} \overline{\mathbf{u}}_{j}^{n+1=}-\frac{\Delta t}{\overline{\Delta x}_{j}^{n}}\left(\overline{\mathbf{f}}^{-}\left(\mathbf{u}_{j}^{n+1=,+}, \mathbf{u}_{j+1}^{n+1=,-}\right)-\overline{\mathbf{f}}^{+}\left(\mathbf{u}_{j-1}^{n+1=,+}, \mathbf{u}_{j}^{n+1=,-}\right)\right) .
$$

Finally, $\overline{\mathbf{u}}_{j}^{n+1}$ is defined from these two approximations setting

$$
\overline{\mathbf{u}}_{j}^{n+1}=\mathbf{u}_{j}^{n}+\frac{1}{2}\left[\left(\overline{\mathbf{u}}_{j}^{n+1=}-\mathbf{u}_{j}^{n}\right)+\left(\overline{\mathbf{u}}_{j}^{n+1-}-\overline{\mathbf{u}}_{j}^{n+1=}\right)\right],
$$

which can be equivalently recast as

$$
\begin{aligned}
\overline{\mathbf{u}}_{j}^{n+1}= & \mathbf{u}_{j}^{n}+\frac{\Delta x-\overline{\Delta x}_{j}^{n}}{2 \overline{\Delta x}_{j}^{n}}\left(\mathbf{u}_{j}^{n}+\overline{\mathbf{u}}_{j}^{n+1=}\right)-\frac{\Delta t}{2 \overline{\Delta x}_{j}^{n}}\left(\overline{\mathbf{f}}^{-}\left(\mathbf{u}_{j}^{n,+}, \mathbf{u}_{j+1}^{n,-}\right)-\overline{\mathbf{f}}^{+}\left(\mathbf{u}_{j-1}^{n,+}, \mathbf{u}_{j}^{n,-}\right)\right) \\
& -\frac{\Delta t}{2 \overline{\Delta x}_{j}^{n}}\left(\overline{\mathbf{f}}^{-}\left(\mathbf{u}_{j}^{n+1=,+}, \mathbf{u}_{j+1}^{n+1=,-}\right)-\overline{\mathbf{f}}^{+}\left(\mathbf{u}_{j-1}^{n+1=,+}, \mathbf{u}_{j}^{n+1=,-}\right)\right) .
\end{aligned}
$$

To conclude this section, note that away from phase transitions, we have $\overline{\Delta x}_{j}^{n}=\Delta x$ and that the numerical fluxes coincide with the ones of the usual Godunov method. So that we get in such a situation

$$
\begin{aligned}
\overline{\mathbf{u}}_{j}^{n+1}= & \mathbf{u}_{j}^{n}-\frac{\Delta t}{2 \Delta x}\left(\mathbf{f}\left(\mathbf{v}_{\mathbf{r}}\left(0 ; \mathbf{u}_{j}^{n,+}, \mathbf{u}_{j+1}^{n,-}\right)\right)-\mathbf{f}\left(\mathbf{v}_{\mathbf{r}}\left(0 ; \mathbf{u}_{j-1}^{n,+}, \mathbf{u}_{j}^{n,-}\right)\right)\right) \\
& -\frac{\Delta t}{2 \Delta x}\left(\mathbf{f}\left(\mathbf{v}_{\mathbf{r}}\left(0 ; \mathbf{u}_{j}^{n+1=,+}, \mathbf{u}_{j+1}^{n+1=,-}\right)\right)-\mathbf{f}\left(\mathbf{v}_{\mathbf{r}}\left(0 ; \mathbf{u}_{j-1}^{n+1=,+}, \mathbf{u}_{j}^{n+1=,-}\right)\right)\right),
\end{aligned}
$$

and the classical method consisting in a RK2 time integration together with a MUSCL reconstruction strategy for the space discretization is recovered. The scheme is then second-order accurate in both space and time in smooth regions.

\section{Numerical experiments}

We now test our algorithm on several Riemann initial data leading to typical solutions of interest, involving phase transitions or not (see also Section 3 above). The various constants involved in the models (free or congested) are taken as follows:

$$
R=1, \quad V=2, \quad V_{f}=1, \quad V_{c}=0.85, \quad Q=0.5, \quad Q^{-}=0.25, \quad Q^{+}=1.5 .
$$

Note that a state in the free phase will be characterized by the value of its density $\rho$, while for a congested state, we will use the density $\rho$ and the flux $f=\rho v_{c}(\rho, q)$ (the value of $q$ is recovered by inverting (5)). The exact and numerical profiles of the density and the speed are systematically plotted, at a final time $T_{f}$ and for a space step $\Delta x$ that will be specified for each test case.

\subsection{The case of a single phase}

In this section, we consider four Riemann initial data located in a single phase (free or congested). More precisely, the left and right states are as follows. 
TRAFFIC FLOW MODELING


FIG. 9. Test A: $\rho$ (left) and $v$ (right).


FIG. 10. Test B: $\rho$ (left) and $v$ (right).
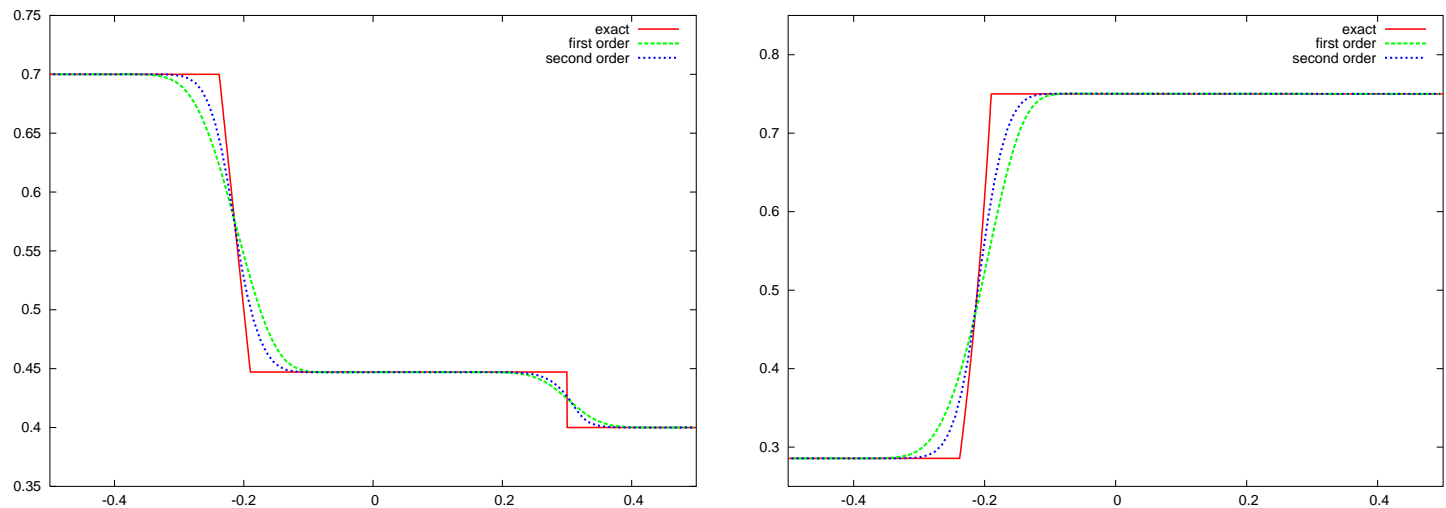

FIG. 11. Test C: $\rho$ (left) and $v$ (right). 



FIG. 12. Test D: $\rho$ (left) and $v$ (right).

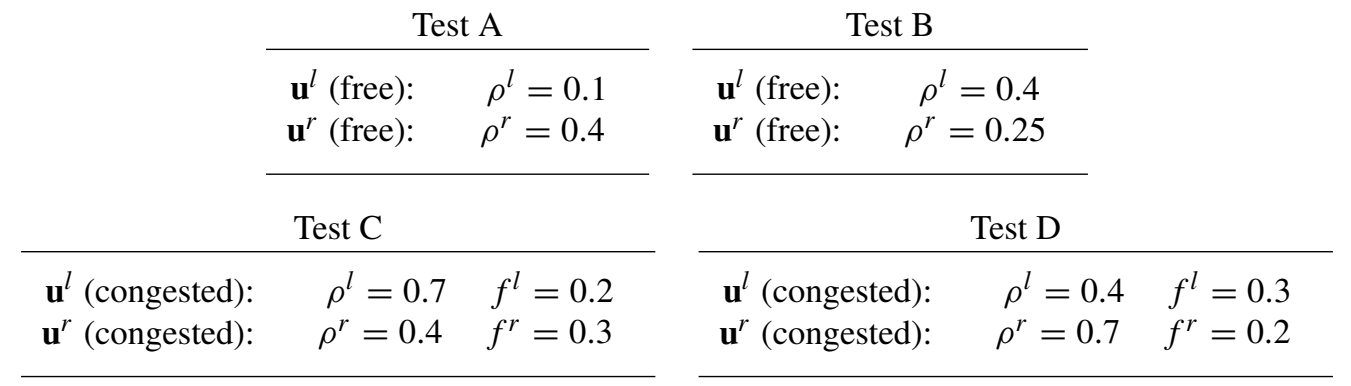

In all these situations, there is no phase transition. Then our method simply reduces to the usual Godunov method since at each interface $x_{j+1 / 2}, j \in \mathbb{Z}$, we necessarily have $\sigma_{j+1 / 2}^{n}=0$. In particular, the method is conservative. In the free phase, the left state is connected directly to the right state, by a shock in Test A (see Figure 9) and a rarefaction in Test B (see Figure 10). In the congested phase, the solution is generally made of two distinct waves. More precisely, we have a rarefaction wave followed by a contact discontinuity in the case of Test C (Figure 11), and a shock wave followed by a contact discontinuity in the case of Test D (Figure 12). Solutions are plotted at time $T_{f}=0.4$ for Tests A, C, and $T_{f}=0.5$ for Tests B, D. The computations have been performed with a mesh containing 100 points per unit interval $(\Delta x=0.01)$. As expected, we observe that the numerical solutions are in good agreement with exact ones.

\subsection{The case of a phase transition from congested to free}

In order to assess the validity of our method, we now consider various situations where a phase transition is present in the solution. We begin with phase transitions from a congested state to a free state.

For Test E, we consider the following left and right states for the Riemann initial data:

Test E

$\begin{array}{rc}\mathbf{u}^{l} \text { (congested): } & \rho^{l}=0.7 \quad f^{l}=0.3 \\ \mathbf{u}^{r} \text { (free): } & \rho^{r}=0.3\end{array}$



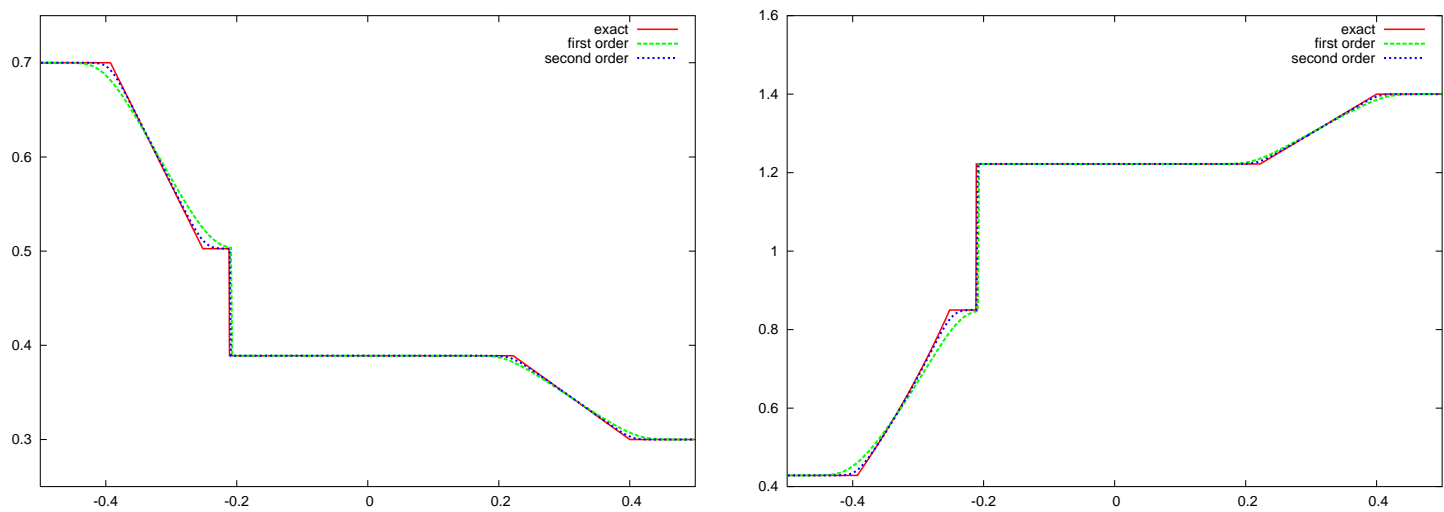

FIG. 13. Test E: $\rho$ (left) and $v$ (right).
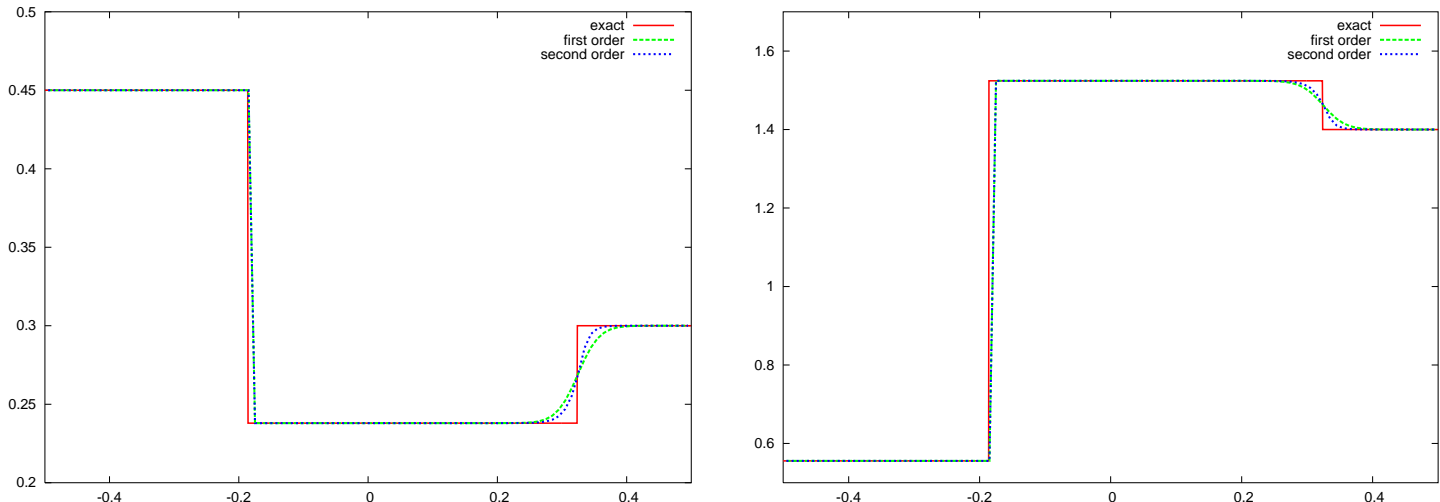

FIG. 14. Test F: $\rho$ (left) and $v$ (right).

leading to a solution made of a rarefaction in the congested phase, followed by a phase transition to a free state, itself followed by a rarefaction wave in the free phase (see Figure 3 , left). The solutions are plotted in Figure 13 at time $T_{f}=0.5$. For this test case, we have used a mesh containing 500 points $(\Delta x=0.002)$.

The next table concerns Test F:

Test F

$\begin{array}{rc}\mathbf{u}^{l} \text { (congested): } & \rho^{l}=0.45 \quad f^{l}=0.25 \\ \mathbf{u}^{r} \text { (free): } & \rho^{r}=0.3\end{array}$

Here, the solution is a shock-like phase transition from the left state to a free state, followed by a shock wave in the free phase (see Figure 3 right). See Figure 14 for the solution at time $T_{f}=0.35$ with $\Delta x=0.01$.

For these two test cases, we observe a very good agreement between the exact and numerical solutions. In particular, we note that the phase transitions we obtain join the right states and 
propagate apparently with the right speed (theoretically given by the Rankine-Hugoniot conditions). This means that the mass conservation property is not lost at the discrete level.

In the case of Test $\mathrm{E}$, we note that the numerical diffusion of the scheme on the rarefaction waves makes the free state of the phase transition hardly apparent at the final time $T_{f}$ proposed. This phenomenon will be even more pronounced in the numerical solutions presented below for Tests H, I.

\subsection{The case of a phase transition from free to congested}

Let us now address the case of phase transitions from a free state to a congested state.

For the Test $\mathrm{G}$, we choose

\begin{tabular}{|c|c|}
\hline & Test G \\
\hline $\mathbf{u}^{l}$ (free): & $\rho^{l}=0.35$ \\
\hline $\mathbf{u}^{r}$ (congested): & $\rho^{r}=0.6 \quad f^{r}=0.25$ \\
\hline
\end{tabular}

The corresponding solution is a shock-like phase transition to a congested state followed by a contact discontinuity (see Figure 4, left). Figure 15 plots the solution at time $T_{f}=0.6$ with $\Delta x=0.002$.
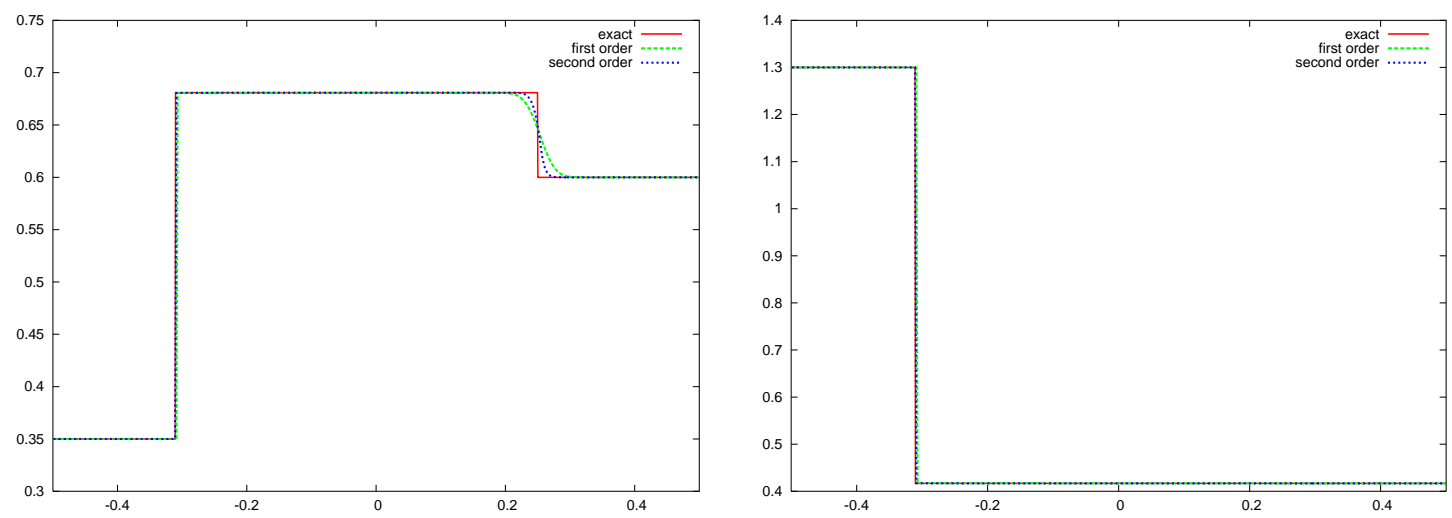

FIG. 15. Test G: $\rho$ (left) and $v$ (right).

For Tests $\mathrm{H}$ and I, we take

Test $\mathrm{H}$

$\begin{array}{r}\mathbf{u}^{l} \text { (free): } \\ \mathbf{u}^{r} \text { (congested): } \quad \rho^{l}=0.24 \\ \hline\end{array}$

Test I

$\mathbf{u}^{l}$ (free):
$\mathbf{u}^{r}$ (congested): $\quad \rho^{l}=0.215$

leading to two solutions composed of a phase transition followed by a rarefaction wave, and a contact discontinuity propagating with a positive speed. In the case of Test $\mathrm{H}$, the "foot" of the rarefaction wave propagates with a speed very close to the one of the phase transition (see Figure 4. right), while for Test I, the rarefaction is simply attached to the transition (see Figure 5, left). These properties make the congested state of the phase transition more difficult to capture properly, due 
to the numerical diffusion of the scheme which is present in the rarefaction wave. Note that this state is always over-estimated from the proposed averaging strategy. However, we observe a good agreement between the numerical solution and the exact solution, and as expected, the numerical solution becomes really better when the order of accuracy of the method is higher, as illustrated in Figures 16 and 17 where $\Delta x=0.0005$ ( $T_{f}=0.8$ for both cases).


FIG. 16. Test H: $\rho$ (left) and $v$ (right).
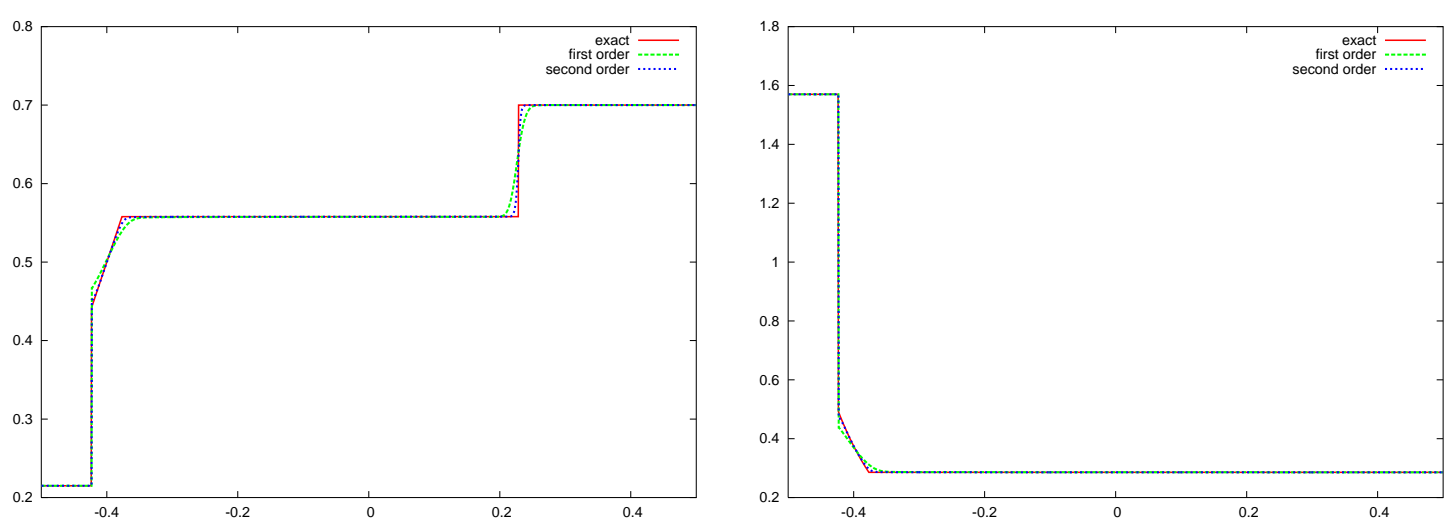

FIG. 17. Test I: $\rho$ (left) and $v$ (right).

Finally, we consider Test $\mathbf{J}$ for which the solution is a phase transition followed by a contact discontinuity (see Figure 5, right). The initial data is such that

Test J

\begin{aligned} \hline $\mathbf{u}^{l}$ (free): & \multicolumn{2}{c}{$\rho^{l}=0.1$} \\ $\mathbf{u}^{r}$ (congested): & $\rho^{r}=0.7 \quad f^{r}=0.2\end{aligned}$

and the solutions are plotted in Figure 18 at time $T_{f}=1.5(\Delta x=0.01)$. 

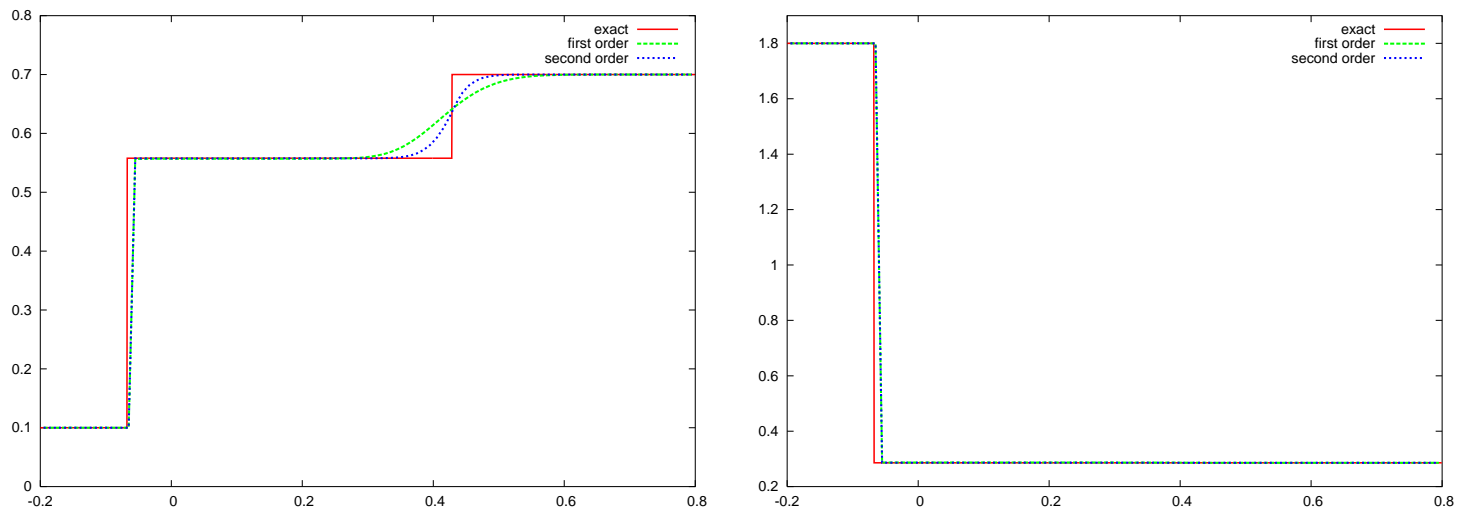

FIG. 18. Test J: $\rho$ (left) and $v$ (right).

We have covered in this section all possible situations described in Section 3 for a Riemann problem. We have observed that the numerical solutions are in accordance with the exact ones and exhibit sharp phase transitions when present (i.e. the corresponding profiles are infinitely thin). This proves the relevance of our approach. In the next section, we propose to estimate the mass conservation errors introduced by the sampling procedure of the scheme. Of course, these are expected to be very small since otherwise the numerical solutions would not be as close as they are to the exact ones. Moreover, we will see that the conservation errors decrease with the mesh size.

\section{Measure of the conservation errors}

Due to the random sampling present in Step 2 (Modified), our method does not strictly conserve the mass $\rho$. We therefore propose to measure the conservation errors on piecewise constant numerical solutions $\rho_{v}$ defined as

$$
\rho_{\nu}(x, t)=\rho_{j}^{n} \quad \text { if }(x, t) \in\left[x_{j-1 / 2}, x_{j+1 / 2}\right) \times\left[t^{n}, t^{n+1}\right),
$$

between times $t=0$ and $t=T$, for some $T>0$. We denote by $\left[x_{0}, x_{1}\right]$ the computational domain and we proceed exactly as in [4]: we compare with 0 the function $E: T \in \mathbb{R}^{+} \rightarrow E(T) \in \mathbb{R}$ with $E(T)$ defined by relation

$$
\begin{aligned}
\int_{x_{0}}^{x_{1}} \rho_{\nu}(x, T) \mathrm{d} x \times E(T)= & \int_{x_{0}}^{x_{1}} \rho_{\nu}(x, T) \mathrm{d} x-\int_{x_{0}}^{x_{1}} \rho_{\nu}(x, 0) \mathrm{d} x \\
& +\int_{0}^{T}\left\{\rho v_{c}(\rho, q)\right\}_{\nu}\left(x_{1}, t\right) \mathrm{d} t-\int_{0}^{T}\left\{\rho v_{c}(\rho, q)\right\}_{\nu}\left(x_{0}, t\right) \mathrm{d} t .
\end{aligned}
$$

Recall that $q=\rho V$ in the free phase. $E(T)$ represents the relative conservation error of $\rho$ at time $T$ on the interval $\left[x_{0}, x_{1}\right]$. In the tables below, we give for Tests E, F, G, H, I, J the values of the $L^{1}$-norm $\frac{1}{T_{f}}\|E\|_{L^{1}\left(0, T_{f}\right)}$ of $E$, namely

$$
\frac{1}{T_{f}}\|E\|_{L^{1}\left(0, T_{f}\right)}=\frac{1}{T_{f}} \int_{0}^{T_{f}}|E(T)| \mathrm{d} T=\sum_{n=0}^{n=T_{f} / \Delta t-1} \frac{t^{n+1}-t^{n}}{T_{f}}\left|E\left(t^{n}\right)\right|,
$$


TABLE 1

Conservation errors (first-order scheme).

\begin{tabular}{ccccccc}
\hline \# of points & Test E & Test F & Test G & Test H & Test I & Test J \\
\hline 100 & $0.44 \%$ & $0.22 \%$ & $0.64 \%$ & $0.39 \%$ & $0.91 \%$ & $0.65 \%$ \\
500 & $0.16 \%$ & $0.11 \%$ & $0.17 \%$ & $0.11 \%$ & $0.22 \%$ & $0.15 \%$ \\
1000 & $0.094 \%$ & $0.075 \%$ & $0.095 \%$ & $0.055 \%$ & $0.11 \%$ & $0.081 \%$ \\
2000 & $0.051 \%$ & $0.039 \%$ & $0.057 \%$ & $0.025 \%$ & $0.052 \%$ & $0.045 \%$ \\
\hline
\end{tabular}

TABLE 2

Conservation errors (second-order scheme).

\begin{tabular}{ccccccc}
\hline \# of points & Test E & Test F & Test G & Test H & Test I & Test J \\
\hline 100 & $0.25 \%$ & $0.26 \%$ & $0.23 \%$ & $0.35 \%$ & $0.87 \%$ & $0.71 \%$ \\
500 & $0.054 \%$ & $0.12 \%$ & $0.071 \%$ & $0.10 \%$ & $0.21 \%$ & $0.19 \%$ \\
1000 & $0.030 \%$ & $0.08 \%$ & $0.044 \%$ & $0.054 \%$ & $0.11 \%$ & $0.11 \%$ \\
2000 & $0.016 \%$ & $0.041 \%$ & $0.031 \%$ & $0.027 \%$ & $0.052 \%$ & $0.05 \%$ \\
\hline
\end{tabular}

where $T_{f}$ is the final time of the corresponding simulations presented in the previous section. We will consider four different meshes containing 100, 500, 1000 and 2000 points per unit interval. The computational domain $\left[x_{0}, x_{1}\right]$ is always $[-0.5,0.5]$ except for Test $\mathbf{J}$ for which it is $[-0.2,0.8]$. Of course, when the solution remains in a single phase (Tests A, B, C, D), our algorithm reduces to the classical Godunov scheme and so is actually conservative. As expected, we observe for the other test cases that the conservation errors are decreasing with the mesh size. We do not note a great improvement when dealing with the second-order scheme. This is not really surprising since conservation errors come from the presence of phase transitions, and the scheme remains first-order accurate near phase transitions.

For the sake of completeness, in the following two tables we give the $L^{1}$-error between the numerical solution and the exact one for all the test cases and several mesh sizes. Note that the $L^{1}$ norm is computed on the whole computational domain, that is, taking into account regions that are

TABLE 3

$L^{1}$-errors (first-order scheme).

\begin{tabular}{cccccc}
\hline \# of points & Test A & Test B & Test C & Test D & Test E \\
\hline 100 & $2.29 e^{-3}$ & $3.22 e^{-3}$ & $7.87 e^{-3}$ & $9.50 e^{-3}$ & $8.64 e^{-3}$ \\
500 & $4.58 e^{-4}$ & $9.87 e^{-4}$ & $3.17 e^{-3}$ & $4.29 e^{-3}$ & $2.99 e^{-3}$ \\
1000 & $2.29 e^{-4}$ & $5.72 e^{-4}$ & $2.08 e^{-3}$ & $3.04 e^{-3}$ & $1.74 e^{-3}$ \\
2000 & $1.14 e^{-4}$ & $3.26 e^{-4}$ & $1.34 e^{-3}$ & $2.15 e^{-3}$ & $1.05 e^{-3}$ \\
\hline \# of points & Test F & Test G & Test H & Test I & Test J \\
\hline 100 & $3.50 e^{-3}$ & $9.67 e^{-3}$ & $9.84 e^{-3}$ & $8.19 e^{-3}$ & $1.18 e^{-2}$ \\
500 & $7.76 e^{-4}$ & $2.03 e^{-3}$ & $4.81^{-3}$ & $4.76 e^{-3}$ & $5.13 e^{-3}$ \\
1000 & $6.05 e^{-4}$ & $1.63 e^{-3}$ & $3.55 e^{-3}$ & $3.27 e^{-3}$ & $2.82 e^{-3}$ \\
2000 & $1.95 e^{-4}$ & $1.01 e^{-3}$ & $2.53 e^{-3}$ & $2.12 e^{-3}$ & $2.13 e^{-3}$ \\
\hline
\end{tabular}


TABLE 4

$L^{1}$-errors (second-order scheme).

\begin{tabular}{cccccc}
\hline \# of points & Test A & Test B & Test C & Test D & Test E \\
\hline 100 & $1.73 e^{-3}$ & $1.07 e^{-3}$ & $4.27 e^{-3}$ & $5.92 e^{-3}$ & $4.18 e^{-3}$ \\
500 & $3.48 e^{-4}$ & $2.16 e^{-4}$ & $1.11 e^{-3}$ & $2.11 e^{-3}$ & $8.60 e^{-4}$ \\
1000 & $1.74 e^{-4}$ & $1.08 e^{-4}$ & $6.10 e^{-4}$ & $1.34 e^{-3}$ & $3.17 e^{-4}$ \\
2000 & $8.69 e^{-5}$ & $5.40 e^{-5}$ & $3.37 e^{-4}$ & $8.52 e^{-4}$ & $2.15 e^{-4}$ \\
\hline \# of points & Test F & Test G & Test H & Test I & Test J \\
\hline 100 & $3.00 e^{-3}$ & $5.12 e^{-3}$ & $7.42 e^{-3}$ & $5.00 e^{-3}$ & $7.98 e^{-3}$ \\
500 & $5.98 e^{-4}$ & $6.19 e^{-4}$ & $2.16 e^{-3}$ & $2.48 e^{-3}$ & $3.02 e^{-3}$ \\
1000 & $5.16 e^{-4}$ & $7.23 e^{-4}$ & $1.25 e^{-3}$ & $1.41 e^{-3}$ & $1.23 e^{-3}$ \\
2000 & $1.49 e^{-4}$ & $4.13 e^{-4}$ & $7.59 e^{-4}$ & $6.97 e^{-4}$ & $7.12 e^{-4}$ \\
\hline
\end{tabular}

possibly not smooth. However, we observe that for each scheme the errors decrease with the mesh size (the convergence seems secured), while the second-order scheme allows obtaining smaller errors as expected.

\section{Acknowledgments}

The authors thank N. Seguin and F. Coquel for useful discussions at the beginning of and during the work. They also thank the referees for their attentive comments.

\section{REFERENCES}

1. Abgrall, R. Generalization of Roe scheme for the computation of mixture of perfect gases. Rech. Aerosp. 6 (1988), 31-43. Zbl 0662.76097

2. Bellomo, N., Coscia, V., \& Delitala, M. On the mathematical theory of vehicular traffic flow I. Fluid dynamic and kinetic modelling. Math. Models Methods Appl. Sci. 12 (2002), 1801-1844. Zbl 1041.76061 MR 1946724

3. Berthon, C. Why the MUSCL-Hancock scheme is $\mathrm{L}^{1}$-stable. Numer. Math. 104 (2006), 27-46. Zbl 1106.65079 MR 2232001

4. Chalons, C. Transport-equilibrium schemes for computing nonclassical shocks. Scalar conservation laws. Numer. Methods Partial Differential Equations 24 (2008), 1127-1147.

5. Chalons, C. Numerical approximation of a macroscopic model of pedestrian flows, SIAM J. Sci. Comput. 29 (2007), 539-555.

6. Chalons, C., \& Coquel, F. Capturing infinitely sharp discrete shock profiles with the Godunov scheme. Proc. 11th International Conference on Hyperbolic Problems: Theory, Numerics, Applications, S. Benzoni-Gavage and D. Serre (eds.), Springer (2008), 363-370.

7. Chalons, C., \& LeFloch, P. G. Computing undercompressive waves with the random choice scheme. Nonclassical shock waves. Interfaces Free Bound. 5 (2003), 129-158. Zbl 1038.35134 MR 1979428

8. Colella, P. Glimm's method for gas dynamics. SIAM J. Sci. Statist. Comput. 3 (1982), 76-110. Zbl 0502.76073 MR 0651869

9. Colombo, R. M. A $2 \times 2$ hyperbolic traffic flow model. Math. Comput. Modelling 35 (2002), 683-688. Zbl 0994.90021 MR 1884026 
10. Colombo, R. M. Hyperbolic phase transitions in traffic flow. SIAM J. Appl. Math. 63 (2002), 708-721. Zbl 1037.35043 MR 1951956

11. Colombo, R. M., Goatin, P., \& Priuli, F. S. Global well posedness of traffic flow models with phase transitions. Nonlinear Anal. Ser. A 66 (2007), 2413-2426. Zbl 1113.35123 MR2312597

12. Drake, J. S., Schofer, J. L., \& MAY, A. D. A statistical analysis of speed density hypothesis. Highway Research Record 154 (1967), 53-87.

13. Goatin, P. The Aw-Rascle vehicular traffic flow model with phase transitions. Math. Comput. Modelling 44 (2006), 287-303. Zbl pre05248498 MR 2239057

14. Godlewsky, E., \& Raviart, P. A. Hyperbolic Systems of Conservation Laws. Ellipse (1991). Zbl 0768.35059 MR 1304494

15. Helbing, D. Traffic and related self-driven many-particle systems. Rev. Modern Phys. 73 (2001), 10671141.

16. Hoff, D. Invariant regions for systems of conservation laws. Trans. Amer. Math. Soc. 289 (1985), 591610. Zbl 0535.35056 MR 0784005

17. Kerner, B. The Physics of Traffic. Springer (2004).

18. LeFloch, P. G. Hyperbolic Systems of Conservation Laws: The theory of classical and nonclassical shock waves. E.T.H. Lecture Notes Ser., Birkhäuser (2002). Zbl 1019.35001 MR 1927887

19. Lighthill, M. J., \& Whitham, G. B. On kinematic waves. II. A theory of traffic flow on long crowded roads. Proc. Roy. Soc. London Ser. A 229 (1955), 317-345. Zbl 0064.20906 MR 0072606

20. Richards, P. I. Shock waves on the highway. Operations Res. 4 (1956), 42-51. MR 0075522

21. TEMPle, B. Systems of conservation laws with coinciding shock and rarefaction curves. Nonlinear Partial Differential Equations (Durham, NH, 1982), Contemp. Math. 17, Amer. Math. Soc. (1983), 143151. Zbl 0538.35050

22. Toro, E. F. Riemann Solvers and Numerical Methods for Fluid Dynamics. Springer (1999). Zbl 0923.76004

23. VAN LEER, B. Towards the ultimate conservative difference scheme. V. A second-order sequel to Godunov's method. J. Comput. Phys. 32 (1979), 101-136. Zbl 0939.76063 MR 1486274

24. Zhong, X.-G., Hou, T. Y., \& LeFloch, P. G. Computational methods for propagating phase boundaries. J. Comput. Phys. 124 (1996), 192-216. Zbl 0855.73080 MR 1378558 